\title{
Six-term exact sequences for smooth generalized crossed products
}

\author{
Olivier Gabriel and Martin Grensing
}

\begin{abstract}
We define smooth generalized crossed products and prove six-term exact sequences of Pimsner-Voiculescu type. This sequence may, in particular, be applied to smooth subalgebras of the quantum Heisenberg manifolds in order to compute the generators of their cyclic cohomology. Further, our results include the known results for smooth crossed products. Our proof is based on a combination of arguments from the setting of (Cuntz-)Pimsner algebras and the Toeplitz proof of Bott periodicity.
\end{abstract}

Mathematics Subject Classification (2010). 46L87, 19K35, 58B34.

Keywords. K-theory, kk-theory, cyclic cohomology, generalized crossed product, Pimsner algebra, quantum Heisenberg manifolds.

\section{Introduction}

In classical bivariant K-theory, the Pimsner-Voiculescu six-term exact sequence (PMV-sequence) [PV80] is one of the main results, allowing to calculate the Ktheory of crossed products in many cases. While proved originally for monovariant K-theory, it can be proved more simply using KK-theory, see [Bla98]. J. Cuntz ([Cun84]) has given a proof that applies, at least partially, to all split exact, homotopy invariant stable functors on the category of $\mathrm{C}^{*}$-algebras with values in the category of Abelian groups. His proof is based on the notion of quasi-homomorphism (see Definition 9). These techniques were later generalized to the setting of locally convex (or even bornological) algebras ([CMR07]). The by now classical Toeplitz proof of Bott periodicity for $\mathrm{C}^{*}$-algebras ([Cun84]) may then be modified to yield a proof of the six-term exact sequence, and J. Cuntz gives in [Cun05] an analogous sequence involving smooth crossed products in the locally convex setting and outlines a proof.

Our result extends the results obtained by R. Nest in [Nes88]. In this article, a six-term exact sequence was established for smooth crossed products by $\mathbb{Z}$, for the particular case of periodic cyclic cohomology and under additional hypotheses on the algebra and the action.

Meanwhile, M. Pimsner presents in [Pim97] a simultaneous generalization of crossed products and Cuntz-Krieger algebras and proves a PMV-type sequence, using 
a certain universal Toeplitz-type $\mathrm{C}^{*}$-algebra. We are ultimately interested in "smooth versions" of the so-called Pimsner algebras. In the $\mathrm{C}^{*}$-setting, these can be described in an essentially equivalent way using generalized crossed products (see [AEE98]). This alternative approach inspired our Section 6. We would like to note that even though we do not refer to [KS97] explicitly, it has to some extent guided our approach.

Our aim is to establish a six-term exact sequence for half-exact, diffotopy-invariant and $\mathbb{K}^{\infty}$-stable functors. The proof is a combination of Pimsner's methods in the $\mathrm{C}^{*}$ setting and Cuntz's techniques introduced in the Toeplitz proof of Bott periodicity in the smooth setting. One of the main results is the "equivalence" of a certain Toeplitztype algebra with a smaller algebra as formulated in Theorem 33. Further, the passage from crossed products to our setting naturally causes difficulties when considering the equivalence between the "base algebra" and a certain ideal inside the Toeplitz-type algebra. It is here that we have to impose further size restrictions on the algebras we start with. We finally apply this to quantum Heisenberg manifolds (QHMs).

The contents of this article are as follow: In Section 2, we introduce some general theory related to locally convex algebras. Section 3 is devoted to a smooth version of Morita equivalence, namely Morita contexts. Quasi-homomorphisms and their properties are introduced in Section 4, while the smooth Toeplitz algebra (in the sense of Cuntz) is defined in Section 5. Both will play essential parts in our construction.

At this point, we are ready to introduce our notion of smooth generalized crossed product (Section 6) and the associated Toeplitz-type algebras (Section 7). A natural extension of locally convex algebras is then given, which involves a kernel and the Toeplitz-type algebra. To get a PMV-sequence, we need to prove that both the kernel and the Toeplitz algebra are "equivalent" to a certain base algebra (compare Theorems 27 and 33). This is taken care of in Sections 8 and 9, respectively. Finally, we assume half-exactness of $H$ and obtain the six-term exact sequence in Section 10.

As an application, we construct in Section 12 six-term exact sequences for the quantum Heisenberg manifolds (QHMs) in kk-theory and cyclic (co)homology that allow to completely determine the cyclic invariants of the QHMs. The $\mathrm{C}^{*}$-QHMs can be considered as generalized crossed products, and therefore long exact sequences are available. When dealing with cyclic theory, one has to stay in the smooth category. We thus show that the smooth QHMs satisfy our growth conditions, and our techniques therefore apply. Consequently, we get Theorem 44 below as a concrete result.

The explicit calculation of the periodic cyclic cohomology of the QHMs, based on our results, is done in another article (see [Gab13]).

Both authors would like to express their gratitude to G. Skandalis for helpful discussions, remarks and advice.

\section{Preliminaries}

We denote the completed projective tensor product by $\otimes_{\pi}$. By a locally convex algebra we will mean a complete locally convex vector space that is at the same time 
a topological algebra, i.e., the multiplication is continuous. Hence if $\mathcal{A}$ is a locally convex algebra, this means that for every continuous seminorm $p$ on $\mathcal{A}$ there is a continuous seminorm $q$ on $\mathcal{A}$ such that

$$
p(a b) \leqslant q(a) q(b)
$$

for all $a, b \in \mathcal{A}$. An $m$-algebra is a locally convex algebra whose topology is defined by submultiplicative seminorms, i.e., we can take $q=p$ in the above inequality.

If $\mathcal{A}$ is a locally convex algebra, we denote by $Z \mathcal{A}$ the locally convex algebra of differentiable functions from $[0,1]$ to $\mathcal{A}$ all of whose derivatives vanish at the endpoints. We denote by $\mathrm{ev}_{t}^{\mathcal{A}}: Z \mathcal{A} \rightarrow \mathcal{A}$ the evaluations in $t \in I=[0,1]$. The smooth suspension $\mathcal{S} \mathcal{A}$ of a locally convex algebra $\mathcal{A}$ is

$$
\mathcal{S} \mathcal{A}=\left\{f \in C^{\infty}([0,1], \mathcal{A}) \mid f^{(k)}(0)=f^{(k)}(1)=0 \text { for all } k \in \mathbb{N}\right\} .
$$

Notice that $\mathcal{S} \mathcal{A}$ is not equal to $Z \mathcal{A}$ since for $\mathcal{S} \mathcal{A}$ we ask that $f(0)=f(1)=0$.

Definition 1. Let $\phi_{0}, \phi_{1}: \mathcal{A} \rightarrow \mathscr{B}$ be homomorphisms of locally convex algebras. A diffotopy between $\phi_{0}$ and $\phi_{1}$ is by definition a homomorphism $\Phi: \mathcal{A} \rightarrow Z \mathscr{B}$ such that $\mathrm{ev}_{i}^{\mathcal{B}} \circ \Phi=\phi_{i}$ for $i=0,1$.

Definition 2. The smooth compact operators are defined as those compact operators $A \in \mathbb{B}\left(\ell^{2}(\mathbb{N})\right)$ such that if $\left(a_{i, j}\right)$ is the representation of $A$ with respect to the standard basis, then for all $m, n \in \mathbb{N}$ :

$$
\sup _{i, j \in \mathbb{N}}(1+i)^{n}(1+j)^{m}\left|a_{i, j}\right|<\infty .
$$

They are topologized by the increasing family of seminorms $\|_{m, n}$ with

$$
\|A\|_{m, n}:=\sum_{i, j}(1+i)^{m}(1+j)^{n}\left|a_{i, j}\right| .
$$

For any locally convex algebra $\mathscr{B}$, the elements of $\mathbb{K}^{\infty} \otimes_{\pi} \mathscr{B}$ are just the matrices with rapidly decreasing coefficients in $\mathscr{B}$ (see [CMR07], Chapter 2, 3.4).

Definition 3. We call split exact sequences the exact sequences

$$
0 \rightarrow \mathcal{A} \rightarrow \mathscr{B} \rightarrow \mathcal{C} \rightarrow 0
$$

in the category of locally convex algebras which admit a splitting by an algebra homomorphism $\mathcal{C} \rightarrow \mathcal{B}$.

We call linearly split exact sequences the exact sequences of locally convex algebras which only admit a splitting by a (continuous) linear map.

Definition 4. A functor $H$ on the category of locally convex algebras with values in Abelian groups is called 
- $\mathbb{K}^{\infty}$-stable if the natural inclusion $\theta: \mathcal{A} \rightarrow \mathbb{K}^{\infty} \otimes_{\pi} \mathcal{A}, a \mapsto e \otimes a$, obtained by any minimal idempotent $e \in \mathbb{K}^{\infty}$ induces an isomorphism

$$
H(\theta): H(\mathcal{A}) \rightarrow H\left(\mathbb{K}^{\infty} \otimes_{\pi} \mathcal{A}\right),
$$

- diffotopy-invariant if $H\left(\mathrm{ev}_{t}^{\mathcal{A}}\right)$ is independent of $t \in[0,1]$ for every locally convex algebra $\mathcal{A}$,

- split exact if for every short exact sequence of locally convex algebras

$$
0 \rightarrow \mathcal{A} \rightarrow \mathscr{B} \rightarrow \mathcal{C} \rightarrow 0
$$

with a splitting by an (algebra) morphism, the sequence

$$
0 \rightarrow H(\mathcal{A}) \rightarrow H(\mathscr{B}) \rightarrow H(\mathscr{C}) \rightarrow 0
$$

is exact.

\section{Morita contexts and split exact functors}

The following definition of Morita context is basically from [Cun05]. We add some isomorphisms to the definitions in [Cun05] in order to make the existence of a Morita context a weaker condition than being isomorphic.

Definition 5. Let $\mathcal{A}$ and $\mathcal{B}$ be locally convex algebras. A Morita context from $\mathcal{A}$ to $\mathcal{B}$ is given by data $\left(\phi, \mathscr{D}, \psi, \xi_{i}, \eta_{i}\right)$, where $\mathscr{D}$ is a locally convex algebra, $\phi: \mathcal{A} \rightarrow \mathscr{D}$, $\psi: \mathscr{B} \rightarrow \mathscr{D}$ are isomorphisms onto subalgebras of $\mathscr{D}$, and sequences $\eta_{i}, \xi_{i}$ in $\mathscr{D}$ such that

(i) $\eta_{i} \phi(\mathcal{A}) \xi_{j} \in \psi(\mathscr{B})$ for all $i, j$;

(ii) $\left(\eta_{i} \phi(a) \xi_{j}\right)_{i j} \in \mathbb{K}^{\infty} \otimes_{\pi} \psi(\mathcal{B})$;

(iii) $\sum \xi_{i} \eta_{i} \phi(a)=\phi(a)$ for all $a \in \mathcal{A}($ convergence in $\phi(\mathcal{A}))$.

With this definition:

- If $\phi: \mathcal{A} \rightarrow \mathscr{B}$ is an isomorphism, then we get a Morita context by introducing the unitization $\mathcal{B}^{+}$of $\mathcal{B}$. Denoting $\iota: \mathcal{B} \rightarrow \mathscr{B}^{+}$the canonical map and $1^{+}$the unit of $\mathcal{B}^{+}$, a Morita context from $\mathcal{A}$ to $\mathcal{B}$ is given by $\left(\iota \circ \phi, \mathcal{B}^{+}, \iota, 1^{+}, 1^{+}\right)$.

- In particular, there is now a canonical Morita context from $\mathcal{A}$ to $\mathcal{A}$ for any locally convex algebra $\mathcal{A}$.

- If $\mathcal{B} \subseteq \mathbb{K}^{\infty} \otimes_{\pi} \mathcal{C}$ is a subalgebra and we call a corner in $\mathcal{B}$ a subalgebra $\mathcal{A} \subseteq \mathscr{B}$ of the form $\sum_{i=0}^{k} e_{0 i} \mathscr{B} \sum_{i=0}^{k} e_{i 0}$, then there is a trivial context from $\mathcal{A}$ to $\mathscr{B}$.

- If $\mathscr{B}$ is row and column-stable $\left(e_{0 i} \mathscr{B}, \mathscr{B} e_{i 0} \subseteq \mathscr{B}\right.$ for all $\left.i\right)$, then there is a context from $\mathscr{B}$ to $\mathcal{A}$, where $\mathcal{B}$ is a subalgebra of $\mathbb{K}^{\infty} \otimes_{\pi} \mathcal{A}$. 
Definition 6. If $H$ is a $\mathbb{K}^{\infty}$-stable functor, then a Morita context from $\mathcal{A}$ to $\mathscr{B}$ induces a $\operatorname{map} \theta: \mathcal{A} \rightarrow \mathbb{K}^{\infty} \otimes_{\pi} \mathscr{B}, a \mapsto\left(\psi^{-1}\left(\eta_{i} \phi(a) \xi_{j}\right)\right)_{i j}$, and a morphism $H(\mathcal{A}) \rightarrow H(\mathscr{B})$ of Abelian groups $H\left(\phi, \mathscr{D}, \psi, \xi_{i}, \eta_{i}\right):=H(\theta)$.

Note that for $a, a^{\prime} \in \mathcal{A}$ we have

$$
\left(\psi^{-1}\left(\eta_{i} \phi(a) \xi_{j}\right)\right)_{i j}\left(\psi^{-1}\left(\eta_{k} \phi\left(a^{\prime}\right) \xi_{l}\right)\right)_{k l}=\left(\psi^{-1}\left(\eta_{i} \phi(a) \sum_{m} \xi_{m} \eta_{m} \phi\left(a^{\prime}\right) \xi_{j}\right)\right)_{i j},
$$

which equals $\theta\left(a a^{\prime}\right)$ by (iii) in the definition of a Morita context. Hence $\theta$ is indeed a homomorphism.

Definition 7. A Morita bicontext between locally convex algebras $\mathcal{A}$ and $\mathscr{B}$ is given by two Morita contexts from $\mathcal{A}$ to $\mathscr{B}$ and $\mathcal{B}$ to $\mathcal{A}$ respectively, of the form $\left(\phi, \mathscr{D}, \psi, \xi_{i}^{\mathcal{A}}, \eta_{i}^{\mathcal{A}}\right)$ and $\left(\psi, \mathscr{D}, \phi, \xi_{i}^{\mathcal{B}}, \eta_{i}^{\mathcal{B}}\right)$ such that

(i) $\phi(\mathcal{A}) \xi_{i}^{\mathcal{A}} \xi_{j}^{\mathcal{B}} \subseteq \phi(\mathcal{A}), \eta_{i}^{\mathscr{B}} \eta_{j}^{\mathcal{A}} \phi(\mathcal{A}) \subseteq \phi(\mathcal{A})$ (left compatibility),

(ii) $\psi \mathscr{B} \xi_{i}^{\mathscr{B}} \xi_{j}^{\mathcal{A}} \subseteq \psi \mathscr{B}, \eta_{i}^{\mathcal{A}} \eta_{j}^{\mathcal{B}} \psi \mathscr{B} \subseteq \psi \mathscr{B}$ (right compatibility).

Theorem 8. Given two Morita contexts as in the above definition, and a diffotopyinvariant, $\mathbb{K}^{\infty}$-stable functor $H$, we have

$H\left(\psi, \mathscr{D}, \phi, \xi_{i}^{\mathcal{B}}, \eta_{i}^{\mathcal{B}}\right) \circ H\left(\phi, \mathscr{D}, \psi, \xi_{i}^{\mathcal{A}}, \eta_{i}^{\mathcal{A}}\right)=\operatorname{id}_{H(\mathcal{A})}$ if they are left compatible, $H\left(\phi, \mathscr{D}, \psi, \xi_{i}^{\mathcal{A}}, \eta_{i}^{\mathcal{A}}\right) \circ H\left(\psi, \mathscr{D}, \phi, \xi_{i}^{\mathcal{B}}, \eta_{i}^{\mathcal{B}}\right)=\mathrm{id}_{H(\mathbb{B})}$ if they are right compatible.

Proof. The proof is an adaptation of the one from [Cun05], Lemma 7.2. Denote the isomorphism $H(\mathcal{A}) \rightarrow H\left(\mathbb{K}^{\infty} \otimes_{\pi} \mathcal{A}\right)$ given by $\mathbb{K}^{\infty}$-stability by $\varepsilon_{\mathcal{A}}$. Then, more precisely, we have to show that

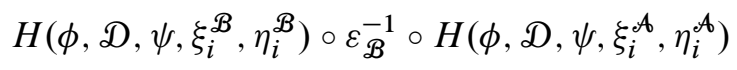

is invertible. We suppose left compatibility; then denoting $\theta_{\mathcal{A}}$ and $\theta_{\mathcal{B}}$ the maps $\mathcal{A} \rightarrow$ $\mathbb{K}^{\infty} \otimes_{\pi} \mathscr{B}$ and $\mathcal{B} \rightarrow \mathbb{K}^{\infty} \otimes_{\pi} \mathcal{A}$ determined by the two contexts, and multiplying by $\varepsilon_{\mathbb{K}} \otimes_{\otimes_{\pi} \mathcal{A}}$ on the left, we see that it suffices to show that the composition $\left(\mathbb{K}^{\infty} \otimes \theta_{\mathscr{B}}\right) \circ \theta_{\mathcal{A}}$ induces an invertible map under $H$. Now this is the map

$$
a \mapsto\left(\phi^{-1}\left(\eta_{i}^{\mathcal{B}}\left(\eta_{k}^{\mathcal{A}} \phi(a) \xi_{l}^{\mathcal{A}}\right) \xi_{j}^{\mathcal{B}}\right)\right)_{i k l j}
$$

and it is diffotopic to the stabilisation as follows. The $(L \times L)$-matrix with entries $\phi^{-1}\left(\hat{\eta}_{\alpha}(t) \phi(a) \hat{\xi}_{\beta}(t)\right), \alpha, \beta \in \mathbb{N}^{2} \cup\{0\}$, with

$$
\begin{array}{ll}
\hat{\xi}_{0}(t)=\cos (t) 1, & \hat{\xi}_{i l}(t)=\sin (t) \xi_{i}^{B} \xi_{l}^{A}, \\
\hat{\eta}_{0}(t)=\cos (t) 1, & \hat{\eta}_{k j}(t)=\sin (t) \eta_{k}^{B} \eta_{j}^{A},
\end{array}
$$

yields a diffotopy of the map in equation (1). 


\section{Quasi-homomorphisms}

We collect some basic properties of quasi-homomorphisms as introduced first in [Cun83], and later adapted to the locally convex setting. See for example [CT06] and [Cun05] for some details.

Definition 9. A quasi-homomorphism between locally convex algebras $\mathcal{A}$ and $\mathcal{B}$ relative to $\widehat{\mathcal{B}}$, denoted by $(\alpha, \bar{\alpha}): \mathcal{A} \rightrightarrows \widehat{\mathcal{B}} \unrhd \mathscr{B}$, is given by a pair $(\alpha, \bar{\alpha})$ of homomorphisms into a locally convex algebra $\widehat{B}$ that contains $\mathscr{B}$ such that $(\alpha-\bar{\alpha})(\mathcal{A}) \subseteq \mathscr{B}$, $\alpha(\mathcal{A}) \mathscr{B} \subseteq \mathscr{B}$ and $\mathscr{B} \alpha(\mathcal{A}) \subseteq \mathscr{B}$.

Note that the definition is symmetric in $\alpha$ and $\bar{\alpha}$. Also, the definition can be generalized, basically by supposing that $\alpha, \bar{\alpha}$ have image in the multipliers of $\mathcal{B}$, but we will not go into this. The use of quasi-homomorphisms as a tool to prove properties of K-theory goes back to [Cun84].

Proposition 10. Let $(\alpha, \bar{\alpha}): \mathcal{A} \rightrightarrows \widehat{\mathcal{B}} \unrhd \mathscr{B}$ be a quasi-homomorphism, $H$ a split exact functor with values in the category of Abelian groups. We have the properties:

(i) $(\alpha, \bar{\alpha})$ induces a morphism, denoted by $H(\alpha, \bar{\alpha})$, from $H(\mathcal{A})$ to $H(\mathscr{B})$.

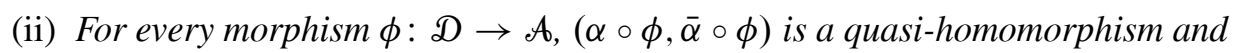

$$
H(\alpha, \bar{\alpha}) \circ H(\phi)=H(\alpha \circ \phi, \bar{\alpha} \circ \phi) .
$$

(iii) If $\phi: \hat{\mathcal{B}} \rightarrow \hat{\mathcal{C}}$ is a morphism and $\mathcal{\complement} \unlhd \hat{\mathcal{C}}$ an ideal such that $(\phi \circ \alpha, \phi \circ \bar{\alpha}): \mathcal{A} \rightrightarrows$ $\hat{e} \unrhd \mathcal{C}$ is a quasi-homomorphism, then $H(\phi \circ \alpha, \phi \circ \bar{\alpha})=H\left(\phi^{\prime}\right) \circ H(\alpha, \bar{\alpha})$, where $\varphi^{\prime}$ is the restriction of $\varphi$ to a morphism $\mathcal{B} \rightarrow \mathcal{C}$.

(iv) If $\mathcal{A}$ is generated as a locally convex algebra by a set $X, \mathfrak{B} \unlhd \widehat{\mathcal{B}}$ an ideal and $\alpha, \bar{\alpha}: \mathcal{A} \rightarrow \widehat{\mathcal{B}}$ are two homomorphisms such that $(\alpha-\bar{\alpha})(X) \subseteq \mathscr{B}$, then $(\alpha, \bar{\alpha})$ is a quasi-homomorphism $\mathcal{A} \rightrightarrows \widehat{B} \unrhd \mathcal{B}$.

(v) If $\alpha-\bar{\alpha}$ is a morphism and orthogonal to $\bar{\alpha}$, then $H(\alpha, \bar{\alpha})=H(\alpha-\bar{\alpha})$.

(vi) If $H$ is diffotopy-invariant, $(\alpha, \bar{\alpha}): \mathcal{A} \rightrightarrows Z \widehat{B} \unrhd Z \mathscr{B}$ a quasi-homomorphism, then the homomorphisms $H\left(\mathrm{ev}_{t} \circ \alpha, \mathrm{ev}_{t} \circ \bar{\alpha}\right)$ are independent of $t \in I$.

Proof. To prove (iv), we let $\mathcal{A}$ be generated by $X$ and $x, x^{\prime} \in X$; then

$$
\alpha\left(x x^{\prime}\right)-\bar{\alpha}\left(x x^{\prime}\right)=\left(\alpha(x)-\bar{\alpha}\left(x^{\prime}\right)\right) \alpha\left(x^{\prime}\right)+\bar{\alpha}\left(x^{\prime}\right)\left(\alpha\left(x^{\prime}\right)-\bar{\alpha}\left(x^{\prime}\right)\right) .
$$

The proof of the other well-known statements can be found for example in [Gre11], Proposition 23.

For subsets $X$ and $Y$ in a locally convex algebra $\mathcal{B}$, we say $Y$ is $X$-stable if $X Y, Y X \subseteq Y$. 
Definition 11. Let $X, Y \subseteq \mathscr{B}$ be subsets. Then $X Y X$ is defined to be the smallest closed $X$-stable (hence locally convex) subalgebra of $\mathscr{B}$ containing $Y$.

The algebra $X Y X$ is obviously independent of the size (but not of the topology) of the ambient algebra: If $\mathscr{B} \subseteq \mathscr{B}^{\prime}$ as a closed subalgebra and $X, Y \subseteq \mathscr{B}$, then it does not matter whether we intersect $X$-stable subalgebras of $\mathscr{B}$ or $\mathscr{B}^{\prime}$. We denote by $X^{+}$the set $X$ with a unit adjoint. We have the following obvious

Lemma 12. $X Y X$ is the locally convex algebra generated by

$$
\left\{\sum_{\text {finite }} x_{i} y_{i} x_{i}^{\prime} \mid x_{i}, x_{i}^{\prime} \in X^{+}, y_{i} \in Y\right\}
$$

and thus depends only on the (complete) locally convex algebras $\mathrm{LC}(X)$ and $\mathrm{LC}(Y)$ generated in $B$ by $Y$ and $X$, respectively:

$$
X Y X=X \operatorname{LC}(Y) X=\operatorname{LC}(X) Y \operatorname{LC}(X) .
$$

Definition 13. For a pair of morphisms $\alpha, \bar{\alpha}: \mathcal{A} \rightarrow \widehat{\mathcal{B}}$ we call the quasi-homomorphism $(\alpha, \bar{\alpha}): \mathcal{A} \rightrightarrows \widehat{\mathscr{B}} \unrhd \mathscr{B}$ with $\mathscr{B}:=\alpha(\mathcal{A})(\alpha-\bar{\alpha})(\mathcal{A}) \alpha(\mathcal{A})$ the associated quasi-homomorphism. We call a quasi-homomorphism minimal if it is associated to $\alpha, \bar{\alpha}: \mathcal{A} \rightarrow \widehat{B}$.

By the above lemma, if $X$ generates $\mathcal{A}$, then $\alpha(X)(\alpha-\bar{\alpha})(X) \alpha(X)=\alpha(\mathcal{A})(\alpha-$ $\bar{\alpha})(\mathcal{A}) \alpha(\mathcal{A})$, using the notation of Lemma 12 above.

\section{The smooth Toeplitz algebra}

We recall the definition and properties of the smooth Toeplitz algebra as introduced in [Cun97], compare Satz 6.1 therein:

Definition 14. The smooth Toeplitz algebra $\mathcal{T}^{\infty}$ is defined by the direct sum $\mathbb{K}^{\infty} \oplus$ $C^{\infty}\left(S^{1}\right)$ of locally convex vector spaces with multiplication induced from the inclusion into the $\mathrm{C}^{*}$-Toeplitz algebra.

It follows from [Cun97] that $\mathcal{T}^{\infty}$ is a nuclear locally convex algebra. We denote by $S$ and $\bar{S}$ the generators of $\mathcal{T}^{\infty}$, set $e:=1-S \bar{S}$, and deduce the following fact from the proof of Lemma 6.2 in [Cun97]:

Lemma 15. There exists a unital diffotopy $\phi_{t}$ such that

$$
\phi_{t}(S)=S(1-e) \otimes 1+f(t)(e \otimes S)+g(t) S e \otimes 1,
$$

where $f, g$ are smooth functions such that $f(0)=0, f(1)=1, g(0)=1$ and $g(1)=0$ and all derivatives of $f$ and $g$ vanish in 0,1 . 
Thus $\phi_{0}: \mathcal{T}^{\infty} \rightarrow \mathcal{T}^{\infty} \otimes_{\pi} \mathcal{T}^{\infty}, x \mapsto x \otimes 1$, is the canonical inclusion into the first variable, and $\phi_{1}$ is the map determined by $S \mapsto \phi_{0}\left(S^{2} \bar{S}\right)+(1-S \bar{S}) \otimes S$.

To unclutter notation, we set $\phi_{0}(x)=x \otimes 1=: \hat{x}$ and $e \otimes x=: \check{x}$ for all $x \in \mathcal{T}^{\infty}$. We also recall that the smooth Toeplitz algebra fits into an extension

$$
0 \rightarrow \mathbb{K}^{\infty} \rightarrow \mathcal{T}^{\infty} \stackrel{\pi}{\rightarrow} C^{\infty}(\mathbb{\mathbb { T }}) \rightarrow 0,
$$

where $C^{\infty}(\mathbb{T})$ is the algebra of smooth functions on the torus. This extension is linearly split by a continuous map $\rho: C^{\infty}(\mathbb{T}) \rightarrow \mathcal{T}^{\infty}$. The ideal is the algebra $\mathbb{K}^{\infty}$ of smooth compact operators, which is isomorphic (as a vector space) to $s \otimes_{\pi} s$, where $s$ is the space of rapidly decreasing sequences ([Cun05]), and hence $\mathbb{K}^{\infty}$ is nuclear. We caution the reader that the smooth compacts are not invariant under conjugation by unitaries if we view them as represented inside some $\mathbb{K}$, where $\mathbb{K}$ denotes the compacts on a separable, infinite-dimensional Hilbert space. They are rapidly decreasing with respect to a choice of base.

Indeed, if $\left(e_{j}\right)$ is the standard basis of $\ell^{2}(\mathbb{N})$, then the orthogonal projections associated to $e_{j}$ are clearly in $\mathbb{K}^{\infty}$. Consider now the vector $u=\sum_{j \geqslant 1} \frac{\alpha}{j} e_{j}$, where $\alpha$ is such that $\|u\|=1$. The associated orthogonal projection is $\left(\frac{\alpha^{2}}{i j}\right)_{i, j}$, which is not in $\mathbb{K}^{\infty}$.

\section{Smooth generalized crossed products}

In the following, a gauge action $\gamma$ on a locally convex algebra $\mathscr{B}$ is an action of $S^{1}$ which is pointwise continuous. In this case, we denote by $\mathscr{B}^{(m)}$ for $m \in \mathbb{Z}$ the space of elements of degree $m$, i.e.,

$$
\mathscr{B}^{(m)}=\left\{b \in \mathscr{B} \mid \gamma_{t}(b)=e^{i 2 \pi m t} b \text { for all } t \in S^{1}\right\} .
$$

We denote the degree 0 part by $\mathcal{A}$ and the degree 1 part by $\mathcal{E}$.

An element $b$ of $\mathscr{B}$ is called gauge smooth if the map $t \mapsto \gamma_{t}(b)$ is smooth.

Definition 16. A smooth generalized crossed product (GCP) $\mathcal{B}$ is a locally convex algebra equipped with an involution and a gauge action such that

- $\mathscr{B}^{(0)}$ and $\mathscr{B}^{(1)}$ generate $\mathscr{B}$ as involutive locally convex algebra;

- all $b \in \mathscr{B}$ are gauge smooth, and the induced map $\mathcal{B} \rightarrow C^{\infty}\left(S^{1} \rightarrow \mathscr{B}\right)$ is continuous.

In the following, we sketch how to compare this definition with the $\mathrm{C}^{*}$-case: Our definition here focusses on the gauge action, thereby remaining close to the notion of generalized crossed product for $\mathrm{C}^{*}$-algebras as given by Abadie, Eilers and Exel in [AEE98]. There is however a way to relate generalized crossed products to the 
so-called Pimsner algebras (see [Pim97]) by using the work of Katsura [Kat03]. The techniques to be used in our proofs are inspired by Pimsner's approach.

The essential difference between the viewpoints of [AEE98] and [Pim97] is the following: Pimsner's construction focusses on the universal properties of the algebra, while [AEE98] emphasises the gauge action. All Pimsner algebras have a gauge action, and taking the degree zero part for this action as base algebra results in a loss of information. More precisely, the data specified for a generalized crossed product suffices to construct a Pimsner algebra which coincides with the generalized crossed product. If we start however with an algebra as constructed by Pimsner, taking the degree zero part as base algebra allows to represent it as a generalized crossed product (this is closely related to the change of the coefficient algebra in Section 3 of [Pim97]).

In a first version of this article [GG10], we had focused on Pimsner's description. The seemingly more intrinsic treatment results in technical complications, and we have found that the additional complexity of the proofs was not justified by the applications we have in mind.

For Pimsner algebras as well as for generalized crossed products, the gauge action is pointwise continuous and therefore we obtain a smooth subalgebra to which our definition applies. For more details and a more general class of examples, see Remark 38 below.

Remark 17. It is easy to see that:

- $\mathcal{A}=\mathscr{B}^{(0)}$ is an involutive subalgebra of $\mathcal{B}$ which we refer to as the base algebra of $\mathscr{B}$.

- $\mathcal{E}=\mathscr{B}^{(1)}$ is an $\mathcal{A}$-bimodule. More generally, $\mathscr{B}^{(m)}$ is an $\mathcal{A}$-bimodule that we write as $\mathcal{E}^{(m)}$.

- If we denote by ${ }^{-}$the involution on $\mathscr{B}$, the expressions $\xi \bar{\eta}$ and $\bar{\xi} \eta$ define " $\mathcal{A}$ valued scalar products" on $\mathcal{E}^{(m)}$.

Notation. In the following we will use the symbols $a$ and $\xi$ as typical notations for elements of $\mathcal{A}$ and $\mathcal{E}$, respectively. To distinguish between $\xi$ as element of the bimodule $\mathcal{E}$ and $\xi$ as element of $\mathscr{B}$, we denote by $S: \mathcal{E} \rightarrow \mathscr{B}$ the inclusion and abbreviate $S(\xi)$ to $S_{\xi}$.

Our final result requires stronger hypotheses. To formulate it, say that a finite sequence $\eta_{1}^{(m)}, \ldots, \eta_{n}^{(m)}$ in $\mathcal{E}^{(m)}$ is a frame for $\mathcal{E}^{(m)}$ if

$$
\sum_{i=1}^{n} \eta_{i}^{(m)} \overline{\eta_{i}^{(m)}}=1
$$

Condition (2) implies in particular that $\mathcal{E}^{(m)}$ is "full" for the "left scalar product", i.e., the span of all the $\xi \bar{\eta}$ is dense in (even equal to) $\mathcal{A}$. Finally, the definition of tame smooth GCP requires some notation: 
Notation. If $\mathscr{B}$ is a smooth GCP with frames $\left(\eta_{i}^{(m)}\right)_{i}$ for every $\mathcal{E}^{(m)}$ with $0 \leqslant m$, we can sort the $\eta_{i}^{(m)}$ according to the lexicographical order of $(i, m)$. We then denote by $\left(\xi_{l}\right)_{l}$ the $\eta_{i}^{(m)}$ numbered according to this order.

For instance, if we have the sequences

$$
\eta_{1}^{(0)}=1 \in \mathcal{A}, \quad\left(\eta_{i}^{(1)}\right)_{i \in\{1,2,3\}}, \quad\left(\eta_{i}^{(2)}\right)_{i \in\{1,2,3\}}, \quad \ldots,
$$

then the sequence $\left(\xi_{l}\right)$ will be

$$
\xi_{1}=1 \in \mathcal{A}, \quad \xi_{2}=\eta_{1}^{(1)}, \quad \xi_{3}=\eta_{2}^{(1)}, \quad \xi_{4}=\eta_{3}^{(1)}, \quad \xi_{5}=\eta_{1}^{(2)}, \quad \xi_{6}=\eta_{2}^{(2)}, \ldots
$$

Notice that with this procedure, $\left(\xi_{l}\right)$ is a sequence which takes its values in $\bigcup_{m \in \mathbb{N}} \mathcal{E}^{(m)}$ and not just in $\mathcal{E}$.

Notation. In order to simplify upcoming calculations we extend the definition of $S_{\xi}$ as follows: If $I \in \mathbb{N}^{n}$ is a multiindex, then we denote by $\xi_{I}$ the $n$-tuple $\left(\xi_{I_{1}}, \ldots, \xi_{I_{n}}\right)$ and set $S_{\xi_{I}}=S_{\xi_{I_{1}}} \ldots S_{\xi_{I_{n}}}, \bar{S}_{\xi_{I}}=\bar{S}_{\xi_{I_{n}}} \ldots \bar{S}_{\xi_{I_{1}}}$. We will refer to $\xi_{I}$ as an $\&$-multivector and write $\left|\xi_{I}\right|:=|I|:=n$.

Definition 18. A tame smooth GCP is a smooth GCP admitting frames in all degrees that satisfy:

(i) there is an integer $N$ such that for any $m \in \mathbb{N}$, the finite sequence $\left(\eta_{i}^{m}\right)_{i}$ has at least 1 and at most $N$ elements;

(ii) for any continuous seminorm $p$ on $\mathscr{B}$, the (real) sequences $\left(p\left(S_{\xi_{l}}\right)\right)_{l}$ and $\left(p\left(\bar{S}_{\xi_{l}}\right)\right)_{l}$ have polynomial growth in $l$.

Remark 19. Two consequences of being tame are: $l / N \leqslant\left|\xi_{l}\right| \leqslant l$ for any $l \in \mathbb{N}$; the (real) sequences $\left(q\left(S^{\left|\xi_{l}\right|}\right)\right)_{l}$ and $\left(q\left(\bar{S}^{\left|\xi_{l}\right|}\right)\right)_{l}$ have polynomial growth in $l$ for any continuous seminorm $q$ on $\mathcal{T}^{\infty}$.

\section{An extension of locally convex algebras}

We assume throughout that $\mathcal{A}$ is unital and complete.

Definition 20. If $\mathscr{B}$ is a smooth GCP, we define $\mathcal{T}_{\mathcal{B}}$, the Toeplitz algebra of $\mathscr{B}$, to be the closed subalgebra of $B \otimes_{\pi} \mathcal{T}^{\infty}$ (completed projective tensor product) generated by

$$
A \mathcal{A} \otimes 1, \quad S(\mathcal{E}) \otimes S, \quad \bar{S}(\mathcal{E}) \otimes \bar{S} .
$$

We write $\mathcal{T}_{\mathscr{B}}^{0}$ for the algebra generated by the same generators, but without closure.

The canonical inclusion will be denoted by $\iota: \mathcal{A} \rightarrow \mathcal{T}_{\mathcal{B}}$. 
Remark 21. In the $\mathrm{C}^{*}$-setting, the analogous construction obtained by taking the $\mathrm{C}^{*}$-tensor product of a Pimsner algebra with the $\mathrm{C}^{*}$-nuclear Toeplitz algebra yields exactly Pimsner's $\mathcal{T}_{E}$ - as can be seen by applying the gauge invariance theorem (see for example [Kat04], Section 6).

Proposition 22. Let $\mathscr{B}$ be a smooth $G C P$ and $\mathcal{T}_{\mathcal{B}}$ the associated Toeplitz algebra. There is a linearly split extension:

$$
0 \rightarrow \operatorname{Ker} \bar{\pi} \rightarrow \mathcal{T}_{\mathcal{B}} \stackrel{\bar{\pi}}{\rightarrow} \mathscr{B} \rightarrow 0 .
$$

Proof. We start from the linearly split exact sequence of locally convex algebras

$$
0 \rightarrow \mathbb{K}^{\infty} \rightarrow \mathcal{T}^{\infty} \stackrel{\pi}{\rightarrow} C^{\infty}(\mathbb{\mathbb { T }}) \rightarrow 0 .
$$

The definition of $\mathcal{T}^{\infty}$ entails that there is a linear splitting $\rho: C^{\infty}(\mathbb{T}) \rightarrow \mathcal{T}^{\infty}$. We can tensor this extension of locally convex algebras by $\mathscr{B}$. Since the initial extension is split, so is the resulting extension:

$$
0 \rightarrow \mathscr{B} \otimes_{\pi} \mathbb{K}^{\infty} \rightarrow \mathscr{B} \otimes_{\pi} \mathcal{T}^{\infty} \rightarrow \mathscr{B} \otimes_{\pi} C^{\infty}(\mathbb{T}) \rightarrow 0 .
$$

Let $\bar{\pi}$ be the restriction of id $\otimes \pi$ to $\mathcal{T}_{\mathcal{B}} \subseteq \mathscr{B} \otimes_{\pi} \mathcal{T}^{\infty}$. This map is continuous as a tensor product of two continuous maps and we get:

$$
0 \rightarrow \operatorname{Ker} \bar{\pi} \rightarrow \mathcal{T}_{\mathscr{B}} \stackrel{\bar{\pi}}{\longrightarrow} \operatorname{Im} \bar{\pi} \rightarrow 0 .
$$

This sequence is linearly split because id $\otimes \rho: \mathscr{B} \otimes_{\pi} C^{\infty}(\mathbb{T}) \rightarrow \mathscr{B} \otimes_{\pi} \mathcal{T}^{\infty}$ restricts to a splitting $\bar{\rho}$ of $\bar{\pi}$ on $\operatorname{Im} \bar{\pi}$.

To see this, denote by $\Delta$ the closed subalgebra of $\mathscr{B} \otimes C^{\infty}(\mathbb{T})$ generated by $a \otimes 1$, $S_{\xi} \otimes U$ and $\bar{S}_{\xi} \otimes \bar{U}$ for $a \in \mathcal{A}$ and $\xi \in \mathcal{E}$. It is clear that $\bar{\pi}\left(\mathcal{T}_{\mathscr{B}}^{0}\right) \subseteq \Delta$. Since $\bar{\pi}$ is continuous and $\Delta$ is closed, we actually get $\bar{\pi}\left(\mathcal{T}_{\mathcal{B}}\right) \subseteq \Delta$.

Using the left and right "scalar products" of Remark 17, any finite product of generators can be written either $S_{\xi_{1}} \ldots S_{\xi_{k}} \otimes U^{k}$ if $k>0$, or $\bar{S}_{\xi_{1}} \ldots \bar{S}_{\xi_{-k}} \otimes \bar{U}^{-k}$ if $k<0$ or $a \otimes 1$ if $k=0$.

In any case, the splitting $\bar{\rho}$ sends this product to an element of $\mathcal{T}_{\mathcal{B}}$ : either $S_{\xi_{1}} \ldots S_{\xi_{k}} \otimes S^{k}$ if $k>0$, or $\bar{S}_{\xi_{1}} \ldots \bar{S}_{\xi_{-k}} \otimes \bar{S}^{-k}$ if $k<0$ or $a \otimes 1$ if $k=0$. Since on the one hand, $\bar{\rho}$ is continuous and $\mathcal{T}_{\mathcal{B}}$ is closed, and on the other hand the finite sums of finite products of generators are dense in $\Delta$ (by definition), it appears that $\bar{\rho}(\Delta) \subseteq \mathcal{T}_{\mathcal{B}}$. Consequently $\Delta=\operatorname{Im} \bar{\pi}$, and in particular, $\operatorname{Im} \bar{\pi}$ is closed.

Let us now prove that $\operatorname{Im} \bar{\pi}$ is isomorphic to $\mathscr{B}$. The restriction of the evaluation map

$$
\text { id } \otimes \mathrm{ev}_{1}: \mathscr{B} \otimes_{\pi} C^{\infty}(\mathbb{T}) \rightarrow \mathscr{B} \otimes_{\pi} \mathbb{C} \simeq \mathscr{B},
$$

where $\mathrm{ev}_{1}(f)=f(1)$, clearly yields a continuous morphism $\operatorname{Im} \bar{\pi} \rightarrow \mathscr{B}$. 
Definition 16 of a smooth GCP entails that $t \mapsto \gamma_{t}(b)$ defines a continuous map $\mathscr{B} \rightarrow C^{\infty}\left(S^{1} \rightarrow \mathscr{B}\right)$. One can identify

$$
C^{\infty}\left(S^{1} \rightarrow \mathscr{B}\right) \simeq \mathscr{B} \otimes_{\pi} C^{\infty}(\mathbb{T})
$$

(see [CST04], p. 62), hence we get a continuous map

$$
\mathscr{B} \rightarrow \mathscr{B} \otimes_{\pi} C^{\infty}(\mathbb{T})
$$

It is readily checked that these maps are inverse to one another. The density of generators hence implies that $\operatorname{Im} \bar{\pi}$ is isomorphic to $\mathscr{B}$ as locally convex algebras.

\section{Morita equivalence of the kernel and base algebras}

Proposition 23. The kernel $\mathcal{C}:=\operatorname{Ker} \bar{\pi}$ of (3) is the locally convex algebra generated by $\mathcal{A} \otimes e, S(\mathcal{E}) \otimes S e$ and $\bar{S}(\mathcal{E}) \otimes e \bar{S}$ in $\mathcal{B} \otimes_{\pi} \mathcal{T}^{\infty}$.

Proof. It is clear that the algebra $\mathcal{C}$ generated by $\mathcal{A} \otimes e, S(\mathcal{E}) \otimes S e$ and $\bar{S}(\mathcal{E}) \otimes e \bar{S}$ is contained the kernel. Since the tensored sequence

$$
0 \rightarrow \mathcal{B} \otimes_{\pi} \mathbb{K}^{\infty} \rightarrow \mathcal{B} \otimes_{\pi} \mathcal{T}^{\infty} \stackrel{\operatorname{id}_{\mathcal{B}} \otimes \pi}{\longrightarrow} \mathcal{B} \otimes_{\pi} C^{\infty}(\mathbb{T}) \rightarrow 0
$$

stays exact, $\operatorname{Ker}(\bar{\pi})=\operatorname{Ker}\left(\mathrm{id}_{\mathcal{B}} \otimes \pi\right) \cap \mathcal{T}_{\mathcal{B}}$.

Now let $x \in \operatorname{Ker}(\bar{\pi}), \varepsilon>0$ and fix a continuous seminorm $p$ on $\mathcal{T}_{\mathscr{B}}$. If $\bar{\rho}$ denotes again the splitting as in the proof of Proposition 22, we can choose a continuous seminorm $q$ such that there is $c_{\bar{\rho}}$ with $p(\bar{\rho}(y)) \leqslant c_{\bar{\rho}} q(y)$, and take a continuous seminorm $p^{\prime}$ on $\mathcal{T}_{\mathscr{B}}$ such that $q\left(\bar{\pi}\left(x^{\prime}\right)\right) \leqslant c_{\bar{\pi}} p^{\prime}\left(x^{\prime}\right)$ for all $x^{\prime}$. As $x \in \mathcal{T}_{\mathcal{B}}$, we may choose a finite sum $x_{0}$ in $\mathcal{T}_{\mathscr{B}}^{0}$ with $p\left(x-x_{0}\right), p^{\prime}\left(x-x_{0}\right)<\varepsilon$. Then $x_{0}-\bar{\rho}\left(\bar{\pi}\left(x_{0}\right)\right) \in$ $\operatorname{Ker}(\bar{\pi})$ and

$$
p\left(x-\left(x_{0}-\bar{\rho}\left(\bar{\pi}\left(x_{0}\right)\right)\right)\right) \leqslant p\left(x-x_{0}\right)+p\left(\bar{\rho}\left(\bar{\pi}\left(x-x_{0}\right)\right)\right) \leqslant\left(1+c_{\bar{\rho}} c_{\bar{\pi}}\right) \varepsilon .
$$

Now $x_{0}-\bar{\rho}\left(\bar{\pi}\left(x_{0}\right)\right)$ is easily seen to be an element in the ideal generated by $1 \otimes e$ in $\mathcal{T}_{\mathscr{B}}$, and thus the other inclusion is proved.

Remark 24. A consequence of this proposition is that $\mathcal{C}$ is included in the algebra $\mathscr{B} \otimes_{\pi} \mathbb{K}^{\infty}$. Every $x \in \mathscr{B} \otimes_{\pi} \mathbb{K}^{\infty}$ (and hence every $x \in \mathcal{C}$ ) has a representation of the form $\sum_{k, l} x_{k, l} \otimes S^{k} e \bar{S}^{l}$, where the $x_{k, l}$ are rapidly decreasing in $k, l$.

To go further in the analysis of the kernel, we rely on the algebra being tame.

Notation. Let $\xi_{i}$ be the sequence of $\mathcal{E}$-multivectors of the Definition 18 . We set

$$
\Xi_{i}:=S_{\xi_{i}} \otimes S^{\left|\xi_{i}\right|} e \quad \text { and } \quad \bar{\Xi}_{i}:=\bar{S}_{\xi_{i}} \otimes e \bar{S}^{\left|\xi_{i}\right|} .
$$


Lemma 25. For any $x \in \mathcal{C}$, the sequence $\left(\bar{\Xi}_{i} x \Xi_{j}\right)_{i, j}$ is rapidly decreasing. More-

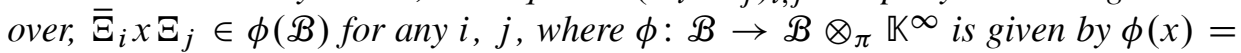
$x \otimes e$.

Proof. Let $x \in \mathcal{C}$ and assume first that $x=\sum_{k, l} x_{k, l} \otimes S^{k} e \bar{S}^{l}$ is a finite sum. Then

$$
\begin{aligned}
\bar{\Xi}_{i} x \Xi_{j} & =\sum_{k, l} \bar{S}_{\xi_{i}} x_{k, l} S_{\xi_{j}} \otimes e \bar{S}\left|\xi_{i}\right| S^{k} e \bar{S}^{l} S^{\left|\xi_{j}\right|} e \\
& =\sum_{k, l} \bar{S}_{\xi_{i}} x_{k, l} S_{\xi_{j}} \otimes \delta_{\left|\xi_{i}\right|, k} \delta_{l,\left|\xi_{j}\right|} e \\
& =\bar{S}_{\xi_{i}} x_{\left|\xi_{i}\right|,\left|\xi_{j}\right|} S_{\xi_{j}} \otimes e,
\end{aligned}
$$

which is clearly an element of $\phi(\mathscr{B})$. This property extends by continuity to $\mathcal{C}$.

We now need to check that $\left(\bar{S}_{\xi_{i}} x_{\left|\xi_{i}\right|,\left|\xi_{j}\right|} S_{\xi_{j}}\right)_{i, j}$ is rapidly decreasing in $i$ and $j$. This can be seen using the fact that $x_{k, l}$ is rapidly decreasing and the polynomial growth of $\bar{S}_{\xi_{i}}, S_{\xi_{j}}$.

More precisely, take a polynomial $R(i, j)$ and fix a continuous seminorm $p$ on $\mathcal{B}$. We prove that $p\left(\bar{S}_{\xi_{i}} x_{\left|\xi_{i}\right|,\left|\xi_{j}\right|} S_{\xi_{j}} R(i, j)\right)$ is bounded above. Since we are considering a tame algebra, $p\left(\bar{S}_{\xi_{i}}\right) p\left(S_{\xi_{j}}\right)$ is bounded by a polynomial $Q_{0}(i, j)$. In turn, we can give a bound for $\left|Q_{0}(i, j) R(i, j)\right|$ by using an "increasing" polynomial $Q(i, j)$. By "increasing", we mean that

$$
i \leqslant i^{\prime} \text { and } j \leqslant j^{\prime} \text { implies that } Q(i, j) \leqslant Q\left(i^{\prime}, j^{\prime}\right) .
$$

Using the relations $\left|X^{p} Y^{q}\right| \leqslant X^{2 p}+Y^{2 q}$, it follows that $\left|Q_{0}(i, j) R(i, j)\right|$ has upper bound $i^{d_{1}}+j^{d_{2}}+K$, for $d_{1}, d_{2}$ and $K$ large enough. Consider now

$$
P(k, l)=\sum_{k^{\prime}, l^{\prime}=1}^{N} Q\left(N(k-1)+k^{\prime}, N(l-1)+l^{\prime}\right) .
$$

This is clearly an increasing polynomial in the above sense.

Since $\left(p\left(x_{k, l}\right)\right)_{k, l}$ is rapidly decreasing in $k, l$, there is an upper bound $C$ such that $p\left(\xi_{k, l}\right) P(k, l) \leqslant C$ for all $k, l \in \mathbb{N}$. Let us estimate

$$
p\left(\bar{S}_{\xi_{i}} x_{\left|\xi_{i}\right|,\left|\xi_{j}\right|} S_{\xi_{j}}\right) \leqslant p\left(x_{\left|\xi_{i}\right|,\left|\xi_{j}\right|}\right) Q(i, j) \leqslant p\left(\xi_{k, l}\right) P\left(k_{0}, l_{0}\right) \leqslant p\left(\xi_{k, l}\right) P(k, l) \leqslant C,
$$

where $k_{0}$ and $l_{0}$ are the integer parts of $i / N$ and $j / N$, respectively, $k=\left|\xi_{i}\right|$ and $l=$ $\left|\xi_{j}\right|$. It follows from Remark 19 that $k_{0} \leqslant k$. By design, $Q(i, j) \leqslant P\left(k_{0}, l_{0}\right)$, which proves the second inequality. $P$ is increasing, and this proves the third inequality.

Lemma 26. If $\mathcal{B}$ is a tame smooth $G C P$, then for any $x \in \mathscr{B} \otimes_{\pi} \mathbb{K}^{\infty}$,

(i) $\sum_{i=1}^{I} \Xi_{i} \bar{\Xi}_{i} x$ is an element of $\mathcal{T}_{\mathscr{B}}$ for any $I \in \mathbb{N}$,

(ii) $\sum_{i=1}^{I} \Xi_{i} \bar{\Xi}_{i} x \stackrel{I \rightarrow \infty}{\longrightarrow} x$ in $\mathcal{T}_{\mathcal{B}}$. 
Proof. Let $x=\sum_{m, n} x_{m, n} \otimes S^{m} e \bar{S}^{n}$. We calculate:

$$
\begin{aligned}
\left(\sum_{i=0}^{I} \Xi_{i} \bar{\Xi}_{i}\right) X & =\sum_{m, n=0}^{\infty} \sum_{i=0}^{I} S_{\xi_{i}} \bar{S}_{\xi_{i}} x_{m, n} \otimes S^{\left|\xi_{i}\right|} e \bar{S}^{\left|\xi_{i}\right|} S^{m} e \bar{S}^{n} \\
& =\sum_{i=0}^{I} \sum_{n=0}^{\infty} S_{\xi_{i}} \bar{S}_{\xi_{i}} x_{\left|\xi_{i}\right|, n} \otimes S^{\left|\xi_{i}\right|} e \bar{S}^{n}
\end{aligned}
$$

which proves that $\sum_{i=1}^{I} \Xi_{i} \bar{\Xi}_{i} X$ is in $\mathcal{T}_{\mathcal{B}}$, as long as the sum converges.

We know that $x_{m, n}$ is rapidly decreasing in $m, n$. We need to show that the sequence $S_{\xi_{i}} \bar{S}_{\xi_{i}} x_{\left|\xi_{i}\right|, n} \otimes S^{\left|\xi_{i}\right|} e \bar{S}^{n}$ is rapidly decreasing $i$ and $n$.

We use the same techniques as in Lemma 25 and fix a continuous seminorm $p$ and a polynomial $R(i, n)$. We can majorise the sequence

$$
\left|R(i, n) p\left(\Xi_{i}\right) p\left(\bar{\Xi}_{i}\right)\right|
$$

by a polynomial $Q(i, n)$, which we can choose increasing in the previous sense (see the proof of Lemma 25).

Since $x_{m, n}$ is rapidly decreasing and $S^{m} e \bar{S}^{n}$ has polynomial growth in $m, n$, the sequence $\left(x_{m, n} \otimes S^{m} e \bar{S}^{n}\right)_{m, n}$ is rapidly decreasing. Considering the polynomial $P(m, n)=\sum_{m^{\prime}=1}^{N} Q\left(N(m-1)+m^{\prime}\right)$, we can find a constant $C$ such that $p\left(x_{m, n} \otimes\right.$ $\left.S^{m} e \bar{S}^{n}\right) P(m, n) \leqslant C$ for any $m, n \in \mathbb{N}$. As a consequence, we get the inequalities

$$
\begin{aligned}
p\left(\Xi_{i} \bar{\Xi}_{i}\left(x_{\left|\xi_{i}\right|, n} \otimes S^{\left|\xi_{i}\right|} e \bar{S}^{n}\right)\right) & \leqslant p\left(x_{\left|\xi_{i}\right|, n} \otimes S^{\left|\xi_{i}\right|} e \bar{S}^{n}\right) P\left(m_{0}, n\right) \\
& \leqslant p\left(x_{m, n}\right) P(m, n) \leqslant C,
\end{aligned}
$$

using the same arguments as in Lemma 25. Therefore, the sequence in $I \in \mathbb{N}$,

$$
\left(\sum_{i=0}^{I} \sum_{n=0}^{\infty} S_{\xi_{i}} \bar{S}_{\xi_{i}} x_{\left|\xi_{i}\right|, n} \otimes S^{\left|\xi_{i}\right|} e \bar{S}^{n}\right)_{I}
$$

is nothing but the partial sums of a summable series. Hence, it admits a finite limit and we will prove that this limit is $x$.

For any degree $m \in \mathbb{N}$, the condition (2) shows that there are two integers $l_{1}$ and $l_{2}$ such that $\sum_{i=l_{1}}^{l_{2}} S_{\xi_{i}} \bar{S}_{\xi_{i}}=1_{\mathcal{A}}$. Algebraically, we see that for any fixed $m$, if $I \geqslant l_{2}$, then

$$
\left(\sum_{i=0}^{I} \Xi_{i} \bar{\Xi}_{i}\right) x_{m, n} \otimes S^{m} e \bar{S}^{n}=x_{m, n} \otimes S^{m} e \bar{S}^{n} .
$$

Hence, for each fixed $m$, we get the identity. The linear forms $\mathcal{C} \rightarrow \mathcal{B}$,

$$
\sum_{m, n} x_{m, n} \otimes S^{m} e \bar{S}^{n} \mapsto x_{m_{0}, n_{0}},
$$

correspond to projections onto entries in the matrix. By evaluating the linear forms on the partial sums, it follows that the (unique) limit is $x$.

Thus, the $\sum_{i=0}^{I} \Xi_{i} \bar{\Xi}_{i}$ form an approximate unit for $\mathcal{C}$. 
Theorem 27. If $\mathcal{B}$ is a tame smooth $G C P$, then there is a Morita bicontext between $\mathcal{C}$ and $\mathcal{A}$. If we denote by $\iota^{\mathcal{C}}$ the inclusion of $\mathcal{A}$ into $\mathcal{C}$ defined by $a \mapsto a \otimes e$, the bicontext is given as follows:

- from $\mathcal{C}$ to $A$ by (id, $\mathcal{C}$, le $, \Xi_{l}, \bar{\Xi}_{l}$ ),

- from $A$ to $\mathcal{C}$ by $(l e, \mathcal{C}, \mathrm{id}, 1 \otimes e, 1 \otimes e)$.

In particular, if $H$ is a $\mathbb{K}^{\infty}$-stable and diffotopy-invariant functor, the canonical inclusion ce: $\mathcal{A} \hookrightarrow \mathcal{C}$ induces an isomorphism $H(\mathcal{A}) \simeq H(\mathcal{C})$.

Proof. Let us first check that we have a Morita context from $\mathcal{C}$ to $\mathcal{A}$, by verifying the conditions of Definition 5: the morphisms clearly are isomorphisms onto subalgebras of $\mathcal{C}$. It remains to check the three conditions of Definition 5. Let $x=\sum_{k, l} x_{k, l} \otimes$ $S^{k} e \bar{S}^{l}$, where $x_{k, l}$ is a homogeneous element of degree $k-l$.

(i) $\bar{\Xi}_{i} X \Xi_{j}$ is in $\iota^{(\mathcal{A}}(\mathcal{A})$ : We saw in Lemma 26 that

$$
\bar{\Xi}_{i}\left(\sum_{k, l} x_{k, l} \otimes S^{k} e \bar{S}^{l}\right) \Xi_{j}=\bar{S}_{\xi_{i}} x_{\left|\xi_{i}\right|,\left|\xi_{j}\right|} \mid S_{\xi_{j}} \otimes e .
$$

Counting the degree proves that $\bar{S}_{\xi_{i}} x_{\left|\xi_{i}\right|,\left|\xi_{j}\right|} S_{\xi_{j}}$ has degree 0 and therefore, it is an

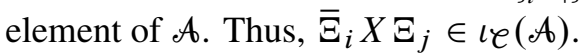

(ii) This is a consequence of Lemma 25.

(iii) This is a consequence of Lemma 26.

It is readily checked that $1 \otimes e$ induces a Morita context:

(i) $1 \otimes e$ is in $\mathcal{C}$, hence $(1 \otimes e) x(1 \otimes e) \in \mathcal{C}$, for any $x \in \mathcal{C}$;

(ii) the sequence is finite and therefore rapidly decreasing;

(iii) $(1 \otimes e)(1 \otimes e)(a \otimes e)=a \otimes e$, thus the partial sums converge in norm.

It is now enough to check that the two Morita contexts are left and right compatible, by checking Definition 7:

(i) This condition is trivially verified, because the underlying algebra is $\mathcal{C}$ itself.

(ii) $(1 \otimes e)\left(S_{\xi_{l}} \otimes S^{\left|\xi_{l}\right|} e\right)$ vanishes if $\left|\xi_{l}\right| \neq 0$. Moreover, the only element with degree 0 is $\xi_{1}=1$ and then $S_{\xi_{1}} \otimes S^{\left|\xi_{1}\right|} e=1 \otimes e$, thus $\iota e(a)(1 \otimes e)=\iota^{e}(a)$. In the same way, $\left(\bar{S}_{\xi_{l}} \otimes e \bar{S}^{\left|\xi_{l}\right|}\right)(1 \otimes e)$ equals either $0\left(\right.$ when $\left.\left|\xi_{l}\right| \neq 1\right)$ or $1 \otimes e$ (when $\left.\left|\xi_{l}\right|=1\right)$ and in this case $(1 \otimes e) \iota \varphi(a)=\iota \varphi(a)$.

\section{Equivalence of the Toeplitz and base algebras}

In this section, we will prove that the smooth Toeplitz algebra $\mathcal{T}_{\mathcal{B}}$ is " $H$-equivalent" to the base algebra $\mathcal{A}$ for any half-exact, $\mathbb{K}^{\infty}$-stable and diffotopy-invariant functor $H$.

For that purpose, we consider a quasi-homomorphism $(\alpha, \bar{\alpha}): \mathcal{T}_{\mathscr{B}} \rightrightarrows \mathscr{B} \otimes \mathcal{T}^{\infty} \unrhd$ $\mathcal{C}$. We will prove that the morphism of Abelian groups $H(\alpha, \bar{\alpha})$ is both left- and rightinvertible - therefore showing that it is invertible. 
We use Theorem 27 to prove that it is right-invertible. It will be more difficult to find a left inverse. We will need to enlarge the image of the quasi-homomorphism. More explicitly, we will have to introduce an algebra $\bar{\varphi}$ and a quasi-homomorphism $(\Phi, \Psi): \mathcal{T}_{\mathscr{B}} \rightrightarrows Z\left(\mathscr{B} \otimes_{\pi} \mathcal{T}^{\infty} \otimes_{\pi} \mathcal{T}^{\infty}\right) \unrhd \overline{\bar{\ell}}$ (Lemma 30), then exhibit a Morita context (Proposition 31) before we can conclude (Theorem 33).

Recall that $\mathcal{C}$ is the subalgebra of $\mathcal{B} \otimes_{\pi} \mathcal{T}^{\infty}$ generated by $\mathcal{A} \otimes e, S(\mathcal{E}) \otimes S e$ and $\bar{S}(\mathcal{E}) \otimes e \bar{S}$.

Lemma 28. Let $\mathcal{B}$ be a smooth GCP. The morphisms $\alpha, \bar{\alpha}: \mathcal{T}_{\mathcal{B}} \rightarrow \otimes_{\pi} \mathcal{T}^{\infty}$ given by

$$
\alpha=\operatorname{id}_{\mathcal{T}_{\mathscr{B}}}, \quad \bar{\alpha}=\operatorname{Ad}(1 \otimes S),
$$

where $\operatorname{Ad}(1 \otimes S)(x)=(1 \otimes S) x(1 \otimes \bar{S})$, define a quasi-homomorphism $(\alpha, \bar{\alpha}): \mathcal{T}_{\mathcal{B}} \rightrightarrows$ $\mathcal{B} \otimes \mathcal{T}^{\infty} \unrhd \mathcal{C}$

Proof. It suffices to see that the ideal $C$ contains $(\alpha-\bar{\alpha})\left(\mathcal{T}_{\mathcal{B}}\right)$. Since

$$
\begin{gathered}
(\alpha-\bar{\alpha})(a \otimes 1)=a \otimes 1-(1 \otimes S)(a \otimes 1)(1 \otimes \bar{S})=a \otimes(1-S \bar{S})=a \otimes e, \\
(\alpha-\bar{\alpha})\left(S_{\xi} \otimes S\right)=S_{\xi} \otimes S-(1 \otimes S)\left(S_{\xi} \otimes S\right)(1 \otimes \bar{S})=S_{\xi} \otimes S e \\
(\alpha-\bar{\alpha})\left(\bar{S}_{\xi} \otimes \bar{S}\right)=\bar{S}_{\xi} \otimes \bar{S}-(1 \otimes S)\left(\bar{S}_{\xi} \otimes \bar{S}\right)(1 \otimes \bar{S})=\bar{S}_{\xi} \otimes e \bar{S}
\end{gathered}
$$

the images of the generators are all in $\ell$, and this suffices by Proposition 10.

Definition 29. If $\mathcal{B}$ is a smooth GCP, we denote by $\bar{\varnothing}$ the closed subalgebra of $\mathcal{B} \otimes_{\pi} \mathbb{K}^{\infty} \otimes_{\pi} \mathcal{T}^{\infty}$ generated by

$$
S_{\xi_{I}} \bar{S}_{\eta_{J}} \otimes S^{p} e \bar{S}^{q} \otimes S^{p^{\prime}} \bar{S}^{q^{\prime}}
$$

where $p, q \in \mathbb{N}, \xi_{I} \in \mathcal{E}^{\left(p+p^{\prime}\right)}$ and $\eta_{J} \in \mathcal{E}^{\left(q+q^{\prime}\right)}$.

We define $\Phi: \mathcal{T}_{\mathcal{B}} \rightarrow Z\left(\mathscr{B} \otimes_{\pi} \mathcal{T}^{\infty} \otimes_{\pi} \mathcal{T}^{\infty}\right)$ to be the restriction of $\operatorname{id}_{\mathcal{B}} \otimes\left(\phi_{t}\right)_{t}$, where $\left(\phi_{t}\right)_{t}: \mathcal{T}^{\infty} \rightarrow Z\left(\mathcal{T}^{\infty} \otimes_{\pi} \mathcal{T}^{\infty}\right)$ is the diffotopy of Lemma 15 , and $\Psi: \mathcal{T}_{\mathscr{B}} \rightarrow$ $Z\left(\mathscr{B} \otimes_{\pi} \mathcal{T}^{\infty} \otimes_{\pi} \mathcal{T}^{\infty}\right)$ to be the restriction of the map $\operatorname{Ad}(1 \otimes \widehat{S})$, independent of $t$, given by $\operatorname{Ad}(1 \otimes \widehat{S})(x)=(1 \otimes \widehat{S})(x \otimes 1)(1 \otimes \widehat{\bar{S}})$ for all $x \in \mathscr{B} \otimes_{\pi} \mathcal{T}^{\infty}$.

Lemma 30. Let $\mathcal{B}$ be a smooth GCP. The morphisms $\Phi, \Psi$ above define a quasihomomorphism $(\Phi, \Psi): \mathcal{T}_{\mathcal{B}} \rightrightarrows Z\left(\mathscr{B} \otimes_{\pi} \mathcal{T}^{\infty} \otimes_{\pi} \mathcal{T}^{\infty}\right) \unrhd Z \overline{\bar{\ell}}$

Proof. We have to check that $(\Phi-\Psi)\left(\mathcal{T}_{\mathcal{B}}\right) \subseteq Z \overline{\mathcal{E}}$ and that this algebra is $\Psi\left(\mathcal{T}_{\mathcal{B}}\right)$ - 
stable. We can check this on the generators for all $t$ by Proposition 10:

$$
\begin{aligned}
\left(\Phi_{t}-\Psi_{t}\right)(a \otimes 1) & =a \otimes \hat{1}-a \otimes \hat{S} \hat{\bar{S}}=a \otimes \hat{e}, \\
\left(\Phi_{t}-\Psi_{t}\right)\left(S_{\xi} \otimes S\right) & =S_{\xi} \otimes(\hat{S}(\hat{1}-\hat{e})+f(t) \check{S}+g(t) \hat{S} \hat{e})-S_{\xi} \otimes \hat{S} \hat{S} \hat{\bar{S}} \\
& =S_{\xi} \otimes(f(t) \check{S}+g(t) \hat{S} \hat{e}), \\
\left(\Phi_{t}-\Psi_{t}\right)\left(\bar{S}_{\xi} \otimes \bar{S}\right) & =\bar{S}_{\xi} \otimes((\hat{1}-\hat{e}) \hat{\bar{S}}+f(t) \check{\bar{S}}+g(t) \hat{\bar{e}} \hat{\bar{S}})-\bar{S}_{\xi} \otimes \hat{S} \hat{\bar{S}} \hat{\bar{S}} \\
& =\bar{S}_{\xi} \otimes(f(t) \check{\bar{S}}+g(t) \hat{\bar{e}}) .
\end{aligned}
$$

All these elements are clearly in $\bar{\ell}$. The $\Psi\left(\mathcal{T}_{\mathcal{B}}\right)$-stability follows from the algebraic relations by an application of Lemma 12 .

Proposition 31. Suppose that $\mathscr{B}$ be a tame smooth GCP. Then there is a Morita context $\left(\mathrm{id}_{\bar{e}}, \overline{\bar{e}}, \iota_{1}, \Xi_{i}^{\prime}, \bar{\Xi}_{j}^{\prime}\right)$ from $\overline{\mathcal{e}}$ to $\mathcal{T}_{\mathcal{B}}$ given by

- the sequences $\Xi_{i}^{\prime}=\Xi_{i} \otimes 1$ and $\bar{\Xi}_{i}^{\prime}=\bar{\Xi}_{i} \otimes 1$, using the notation on $p .510$,

- the morphism $\iota_{1}: \mathcal{T}_{\mathscr{B}} \rightarrow \bar{\ell}$ defined by

$\iota_{1}(a \otimes 1)=a \otimes e \otimes 1, \quad \iota_{1}\left(S_{\xi} \otimes S\right)=S_{\xi} \otimes e \otimes S, \iota_{1}\left(\bar{S}_{\xi} \otimes \bar{S}\right)=\bar{S}_{\xi} \otimes e \otimes \bar{S}$ for $a \in \mathcal{A}$ and $\xi \in \mathcal{E}$.

Notice that the Morita context in fact induces a morphism from $\bar{C}$ to $\iota_{1}\left(\mathcal{T}_{\mathcal{B}}\right) \otimes \mathbb{K}^{\infty}$. We identify $\iota_{1}\left(\mathcal{T}_{\mathcal{B}}\right) \simeq \mathcal{T}_{\mathscr{B}}$ to obtain a map $\theta: \overline{\mathcal{C}} \rightarrow \mathcal{T}_{\mathcal{B}} \otimes_{\pi} \mathbb{K}^{\infty}$.

Proof. We check the conditions of Definition 5.

(i) Algebraically, if we take $Y=S_{\xi_{I}} \bar{S}_{\xi_{J}} \otimes S^{p} e S^{q} \otimes S^{p^{\prime}} S^{q^{\prime}}$ as in Definition 29,

$$
\begin{aligned}
\bar{\Xi}_{i}^{\prime} Y \Xi_{j}^{\prime} & =\left(\bar{S}_{\xi_{i}} \otimes e \bar{S}^{\left|\xi_{i}\right|} \otimes 1\right)\left(S_{\xi_{I}} \bar{S}_{\eta_{J}} \otimes S^{p} e S^{q} \otimes S^{p^{\prime}} S^{q^{\prime}}\right)\left(S_{\xi_{j}} \otimes S^{\left|\xi_{j}\right|} e \otimes 1\right) \\
& =\delta_{\left|\xi_{i}\right|, p} \delta_{\left|\xi_{j}\right|, q} \bar{S}_{\xi_{i}} S_{\xi_{I}} \bar{S}_{\eta_{J}} S_{\xi_{j}} \otimes e \otimes S^{p^{\prime}} \bar{S}^{q^{\prime}} .
\end{aligned}
$$

A simple computation of degree shows that $\bar{\Xi}_{i}^{\prime} Y \Xi_{i}^{\prime}$ is in $\iota_{1}\left(\mathcal{T}_{\mathcal{B}}\right)$.

(ii) Since $\overline{\mathscr{C}}$ is a subalgebra of $\mathscr{B} \otimes_{\pi} \mathbb{K}^{\infty} \otimes_{\pi} \mathcal{T}^{\infty}$, by using the canonical isomorphism with $\left(\mathscr{B} \otimes_{\pi} \mathcal{T}^{\infty}\right) \otimes_{\pi} \mathbb{K}^{\infty}$ all the elements of $\overline{\mathcal{C}}$ can be written as

$$
\sum_{k, l} Y_{k, l} \otimes S^{k} e \bar{S}^{l}
$$

where $\left(Y_{k, l}\right)_{k, l}$ is a rapidly decreasing sequence of $\mathscr{B} \otimes_{\pi} \mathcal{T}^{\infty}$. In the same way as in Lemma 25 , the sequences $\bar{\Xi}_{i}^{\prime}$ and $\Xi_{j}^{\prime}$ have polynomial growth in $i, j$. The sequence $S^{k} e \bar{S}^{l}$ also have polynomial growth. The rapidly decreasing sequence $Y_{k, l}$ can therefore "absorb" these terms and $\bar{\Xi}_{i}^{\prime} Y \Xi_{j}^{\prime}$ is a rapidly decreasing sequence of $B \otimes_{\pi} \mathcal{T}^{\infty}$. 
(iii) Algebraically,

$$
\begin{aligned}
\Xi_{i}^{\prime} \bar{\Xi}_{i}^{\prime} Y & =\left(S_{\xi_{i}} \bar{S}_{\xi_{i}} \otimes S^{\left|\xi_{i}\right|} e \bar{S}^{\left|\xi_{i}\right|} \otimes 1\right)\left(S_{\xi_{I}} \bar{S}_{\eta_{J}} \otimes S^{p} e S^{q} \otimes S^{p^{\prime}} S^{q^{\prime}}\right) \\
& =\delta_{\left|\xi_{i}\right|, p} S_{\xi_{i}} \bar{S}_{\xi_{i}} S_{\xi_{I}} \bar{S}_{\eta_{J}} \otimes S^{p} e S^{q} \otimes S^{p^{\prime}} \bar{S}^{q^{\prime}}
\end{aligned}
$$

and equation (2) proves that we only have to sum the $\xi_{i}$ such that $\left|\xi_{i}\right|=p$ to "reconstruct" $Y$. Just like before, we can start from elements of $\bar{\varphi}$ of the form (4) with a rapidly decreasing $Y_{k, l}$. The end of the argument is very similar to that of Lemma 26: The sequence $\Xi_{i}^{\prime} \bar{\Xi}_{i}^{\prime} Y_{\left|\xi_{i}\right|, l}$ is rapidly decreasing and therefore summable, and then we prove that the limit can only be $\sum_{k, l} Y_{k, l} \otimes S^{k} e \bar{S}^{l}$, by projecting onto the coefficients of this matrix.

We define a morphism $\sigma: \mathcal{T}_{\mathscr{B}} \rightarrow \mathcal{T}_{\mathcal{B}} \otimes_{\pi} \mathbb{K}^{\infty}$ for $x \in \mathcal{T}_{\mathscr{B}}$ by

$$
\sigma(x)=x \otimes e
$$

where we identify $\mathbb{K}^{\infty}$ with the algebra generated by $S^{p} e \bar{S}^{q}$ for $p, q \in \mathbb{N}$, just as in Definition 14.

Lemma 32. If $\mathcal{B}$ is a tame smooth $G C P$ and if $H$ is a $\mathbb{K}^{\infty}$-stable and split exact functor, then

$$
H(\theta) \circ H\left(\Phi_{1}, \Psi_{1}\right)=H(\sigma),
$$

up to identification of $\iota_{1}\left(\mathcal{T}_{\mathcal{B}}\right)$ with $\mathcal{T}_{\mathcal{B}}$. Consequently, $H\left(\Phi_{1}, \Psi_{1}\right)$ is left-invertible.

Proof. Since $\phi_{t}$ is unital, we have

$$
\begin{gathered}
\Phi_{1}(a \otimes 1)=a \otimes 1 \otimes 1=(\operatorname{Ad}(1 \otimes \widehat{S})(a \otimes \hat{1}))+(a \otimes \check{1}), \\
\Phi_{1}\left(S_{\xi} \otimes S\right)=S_{\xi} \otimes\left(\widehat{S}^{2} \hat{\bar{S}}+\check{S}\right)=\left(\operatorname{Ad}(1 \otimes \widehat{S})\left(S_{\xi} \otimes \widehat{S}\right)\right)+\left(S_{\xi} \otimes \check{S}\right) .
\end{gathered}
$$

If we use the notation $\iota_{1}$ introduced in Proposition 31 , we see that $\operatorname{Im}(\operatorname{Ad}(1 \otimes \widehat{S}))$ and $\operatorname{Im}\left(\iota_{1}\right)$ are orthogonal because $\bar{S} e=0=e S$. We can therefore apply Proposition 10 and deduce that

$$
H\left(\Phi_{1}, \Psi_{1}\right)=H\left(\operatorname{Ad}(1 \otimes \widehat{S}) \oplus \iota_{1}, \operatorname{Ad}(1 \otimes \widehat{S})\right)=H\left(\iota_{1}\right)
$$

Using Theorem 27, it is easy to show that $\theta \circ \iota_{1}$ is equal to $\sigma$, up to identification of $\iota_{1}\left(\mathcal{T}_{\mathscr{B}}\right)$ with $\mathcal{T}_{\mathscr{B}}$. Since $H$ is $\mathbb{K}^{\infty}$-stable, $H(\sigma)$ is an isomorphism, which completes the proof.

Let us recall that $\iota: \mathcal{A} \rightarrow \mathcal{T}_{\mathcal{B}}$ is the canonical inclusion, while the injection เe $: \mathcal{A} \rightarrow \mathcal{C}$ was defined in Theorem 27 and the quasi-homomorphism $(\alpha, \bar{\alpha}): \mathcal{T}_{\mathcal{B}} \rightrightarrows$ $\mathscr{B} \otimes \mathcal{T}^{\infty} \unrhd \mathcal{C}$ in Lemma 28. 
Theorem 33. Let $H$ be a split-exact, diffotopy-invariant and $\mathbb{K}^{\infty}$-stable functor from the category of locally convex algebras to that of Abelian groups and $\mathbb{B}$ a tame smooth GCP. The inclusion $\iota: A \mathcal{A} \hookrightarrow \mathcal{T}_{\mathcal{B}}$ induces an isomorphism

$$
H(\iota): H(\mathcal{A}) \stackrel{\sim}{\longrightarrow} H\left(\mathcal{T}_{\mathcal{B}}\right) .
$$

Proof. Let $y:=H(\alpha, \bar{\alpha})$. It suffices to show that $y$ is invertible. Indeed, as

$$
y \circ H(\iota)=H(\alpha \circ \iota, \bar{\alpha} \circ \iota)=H\left(\iota_{C}\right)
$$

and $H\left(\iota_{C}\right)$ is invertible by Theorem 27, so is $H(\iota)$.

It further suffices to show that $y$ is right- and left-invertible. To see rightinvertibility, we use Proposition 10 to see that

$$
H(\alpha, \bar{\alpha}) \circ H(\iota)=H(\alpha \circ \iota, \bar{\alpha} \circ \iota)=H\left(\iota_{C}\right),
$$

and thus $H\left(\iota_{C}\right)$ is invertible by Theorem 27.

To see left-invertibility, we show that there exists a morphism $z$ such that $z \circ y$ is left-invertible. For $z$ we may take the morphism induced by the restriction of $\Phi_{0}$ to a morphism $\mathcal{C} \rightarrow \bar{\ell}$. In other words, we simply view $(\alpha, \bar{\alpha})$ as a quasi-homomorphism into a larger algebra, namely as the quasi-homomorphism $\left(\Phi_{0}, \Psi_{0}\right)$. Since by Lemma $32, H\left(\Phi_{1}, \Psi_{1}\right)$ is invertible and $H$ is diffotopy-invariant, we get the result.

\section{Six-term exact sequence and Chern character}

We now use the theory kk to show that there are exact sequences in kk and bivariant cyclic theory that are compatible with the Chern character and the boundary maps. In particular, we get, specializing the first algebra to $\mathbb{C}$, such sequences in K-theory and cyclic theory.

However, to give our first result, we do not really need kk-theory, but we obtain a six-term exact sequence for every half-exact, $\mathbb{K}^{\infty}$-stable and diffotopy-invariant functor.

Definition 34. A functor $H$ on the category of locally convex algebras with values in Abelian groups is called half-exact if for every linearly split short exact sequence

$$
0 \rightarrow \mathcal{A} \rightarrow \mathscr{B} \rightarrow \mathcal{C} \rightarrow 0
$$

the sequence

$$
H(\mathcal{A}) \rightarrow H(\mathscr{B}) \rightarrow H(\mathcal{C})
$$

obtained by applying $H$ is exact.

It is easy to see that a half-exact functor is always split-exact. These functors are interesting because they yield six-term exact sequences, as we will see shortly. We remind the reader that $\mathcal{S} \mathscr{A}$ denotes the smooth suspension of the algebra $\mathcal{A}$. 
Lemma 35. If $\mathcal{A}, \mathcal{B}$ and $\mathcal{C}$ are locally convex algebras and if $H$ is a half-exact, diffotopy-invariant and $\mathbb{K}^{\infty}$-stable functor, then the extension

$$
0 \rightarrow \mathcal{A} \rightarrow \mathscr{B} \rightarrow \mathcal{C} \rightarrow 0
$$

induces a six-term exact sequence:

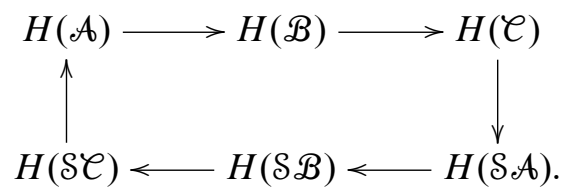

Proof. This is a well-known result (see for instance [CST04], p. 48). One extends the half-exact functor into a homology theory $H_{n}$ by setting $H_{n}(\mathcal{A})=H\left(\mathcal{S}^{n} A\right)$. Observe that $H_{n}$ is automatically Bott periodic, either by carrying over the proof from [Cun05] or by universality of kk; hence the result follows.

Theorem 36. If $\mathcal{B}$ is a tame smooth GCP, then for every half-exact, diffotopy-invariant and $\mathbb{K}^{\infty}$-stable functor $H$ there is a six-term exact sequence

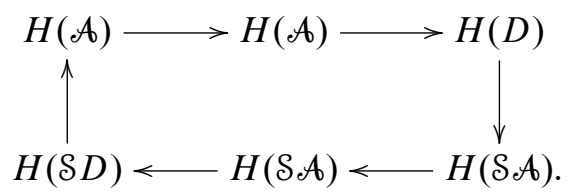

Proof. We just have to combine Lemma 35 with extension (3) and Theorems 27 and 33 to get the six-term exact sequence.

We will now use the version of kk for $m$-algebras introduced in [Cun97]. We also refer to this article for the definition of the bivariant Chern-Connes character $\mathrm{kk}_{*} \rightarrow \mathrm{HP}_{*}$ (which essentially exists by universality of kk).

Theorem 37. Let $\mathcal{B}$ be a smooth GCP. Suppose that $\mathcal{B}$ is an $m$-algebra, set $\mathcal{A}:=\mathscr{B}^{(0)}$ and assume that the Chern-Connes character yields an isomorphism

$$
\mathrm{kk}_{*}(\mathcal{D}, \mathcal{A}) \otimes \mathbb{C} \rightarrow \mathrm{HP}_{*}(\mathscr{D}, \mathcal{A}) \otimes \mathbb{C}
$$

Then

$$
\mathrm{kk}_{*}(\mathscr{D}, \mathscr{B}) \otimes \mathbb{C} \rightarrow \mathrm{HP}_{*}(\mathcal{D}, \mathscr{B}) \otimes \mathbb{C}
$$

is an isomorphism.

In particular, this holds for any $\mathcal{B}$ with commutative base $\mathcal{A}$. 
Proof. This follows by an application of the Five Lemma to

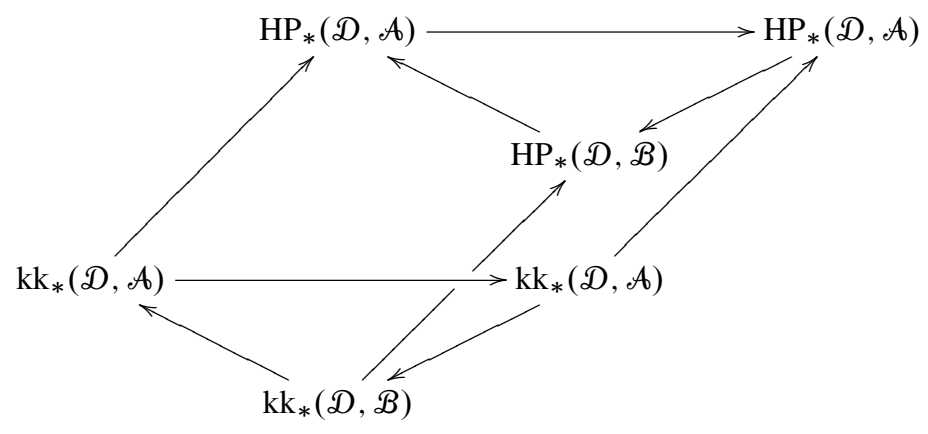

where the maps between kk and cyclic theory denote the bivariant Chern-Connes character from [Cun97], the others come from the long exact sequence.

\section{Application to smooth algebras and crossed products}

Let us start by noting that a Lie group action on a Pimsner algebra induces a smooth GCP:

Remark 38. If $\alpha: G \curvearrowright B$ is a pointwise continuous Lie group action on a $\mathrm{C}^{*}$ Pimsner algebra $B$ which preserves the degree of homogeneous elements, we can extend it to an action $\tilde{\alpha}$ of $\widetilde{G}=G \times S^{1}$ by adding the gauge action to it. In this case, the algebra $\mathcal{B}$ of $\widetilde{G}$-smooth elements of $B$ is a natural smooth subalgebra of $B$. The Lie algebra $\tilde{\mathfrak{g}}$ associated to $\widetilde{G}$ acts by derivations on $\mathscr{B}$.

Moreover, $\mathscr{B}$ is a holomorphically closed Fréchet subalgebra $\mathscr{B}$ of $B$, equipped with the seminorms induced by the action of the universal enveloping algebra $U(\widetilde{G})$ of $\widetilde{G}$ (see for instance [VFGB00], Proposition 3.45). All $b \in \mathscr{B}$ are gauge smooth by construction, and it is then easy to check that the map $\mathscr{B} \rightarrow C^{\infty}\left(S^{1} \rightarrow \mathscr{B}\right)$ is continuous. Hence $\mathscr{B}$ is a smooth GCP.

To link our construction with previous work by Nest ([Nes88]), we prove the following.

Proposition 39. If $\mathcal{A} \hat{x}_{\alpha} \mathbb{Z}$ is a smooth crossed product in the sense of Definition 1.2 of [Nes88], then $\mathcal{A} \hat{x}_{\alpha} \mathbb{Z}$ is a tame smooth GCP in the sense of Definition 16.

Proof. Set $\underline{\mathcal{A}}=\mathcal{A} \hat{x}_{\alpha} \mathbb{Z}$. We start by proving that $\underline{\mathcal{A}}$ is a smooth GCP, using the notations of [Nes88]. We define a gauge action by

$$
\gamma_{t}\left(\sum_{n} a_{n} U^{n}\right)=\sum_{n} e^{i n t} a_{n} U^{n} .
$$


It is clear that $\underline{\mathcal{A}}^{(m)}=\mathcal{A} U^{m}$. Hence, $\underline{\mathcal{A}}^{(0)}=\mathcal{A}$ and $\underline{\mathcal{A}}^{(1)}=\mathcal{A} U$. Clearly, $\underline{\mathcal{A}}^{(0)}$ and $U$ generate $\underline{\mathcal{A}}$ as involutive locally convex algebra.

All elements in $\underline{\mathcal{A}}$ are gauge smooth: indeed an infinitesimal generator $\partial$ a gauge action is given by

$$
\partial\left(\sum_{n} a_{n} U^{n}\right)=\sum_{n}(i 2 \pi n) a_{n} U^{n} .
$$

Seminorms defining the topology on $\underline{\mathcal{A}}$ are

$$
\left\|\sum_{n} a_{n} U^{n}\right\|_{k}=\sup _{n} \rho_{k}(n)\left\|a_{n}\right\|_{k},
$$

where the $\rho_{k}(n)$ are defined in section 1 of [Nes88]. The item (1) of Lemma 1.1 of [Nes88] shows that $|n| \rho_{k}(n) \leqslant \rho_{k+1}(n)$. As a consequence,

$$
\begin{aligned}
\left\|\partial\left(\sum_{n} a_{n} U^{n}\right)\right\|_{k} & =|i 2 \pi| \sup _{n} \rho_{k}(n)|n|\left\|a_{n}\right\|_{k} \\
& \leqslant 2 \pi \sup _{n} \rho_{k+1}(n)\left\|a_{n}\right\|_{k+1} \\
& \leqslant|2 \pi|\left\|\partial\left(\sum_{n} a_{n} U^{n}\right)\right\|_{k+1},
\end{aligned}
$$

because the sequence of seminorms $\|\cdot\|_{k}$ is increasing. Hence, for any $\sum_{n} a_{n} U^{n}$ in $\underline{\mathcal{A}}, \partial\left(\sum_{n} a_{n} U^{n}\right)$ is also in $\underline{\mathcal{A}}$ and $\sum_{n} a_{n} U^{n}$ is gauge smooth.

It is obvious from the definition of the seminorms on $\mathcal{A}$ that the gauge action is isometric. Hence, the topology of $C^{\infty}\left(S^{1} \rightarrow \underline{\mathcal{A}}\right)$ is generated by the seminorms

$$
\sum_{n} a_{n} U^{n} \mapsto\left\|\partial^{l}\left(\sum_{n} a_{n} U^{n}\right)\right\|_{k}
$$

for $k=1,2, \ldots$ and $l=0,1, \ldots$ By iterating the inequality (5), it is therefore easy to prove that the map $\underline{\mathcal{A}} \rightarrow C^{\infty}\left(S^{1} \rightarrow \underline{\mathcal{A}}\right)$ induced by the gauge action is continuous.

We now prove that $\mathcal{A}$ is tame. It is clear that for any $m, U^{m}$ provides a frame for $\underline{\mathcal{A}}^{(m)}$. Hence the sequence $\left(\xi_{i}\right)_{i}=\left(U^{i}\right)_{i}$ satisfies condition (i) of Definition 18.

\section{Application to quantum Heisenberg manifolds}

Quantum Heisenberg manifolds were initially defined by Rieffel in [Rie89]. Here we use the definition of [AEE98] (see Example 3.3).

Definition 40. Given two real numbers $\mu, v$ and an integer $c>0$, we define the quantum Heisenberg manifolds (QHMs or $D_{\mu, \nu}^{c}$ ) to be the $\mathrm{C}^{*}$-completion of the algebra

$$
D_{0}=\left\{F \in C_{\mathrm{c}}\left(\mathbb{Z} \rightarrow C_{\mathrm{b}} b\left(\mathbb{R} \times S^{1}\right)\right) \mid F(p, x+1, y)=e(-c p(y-p v)) F(p, x, y)\right\},
$$


where $e(x)=e^{2 \pi i x}$, equipped with the multiplication

$$
\left(F_{1} \cdot F_{2}\right)(p, x, y)=\sum_{q \in \mathbb{Z}} F_{1}(q, x, y) F_{2}(p-q, x-q 2 \mu, y-q 2 v) .
$$

In the following, we will write $D$ instead of $D_{\mu, \nu}^{c}$ whenever there is no risk of confusion. One can prove that the involution is

$$
F^{*}(p, x, y)=\bar{F}(-p, x-2 p \mu, y-2 p v) .
$$

We recall that

$$
D^{(n)}=\left\{F(p, x, y)=\delta_{n, p} f(x, y) \mid f(x+1, y)=e(-c n(y-n v)) f(x, y)\right\} .
$$

Lemma 41. Given $(c, \mu, v) \in \mathbb{N}^{*} \times \mathbb{R}^{2}$ and an integer $n \in \mathbb{Z}$, there is a frame of two elements $\xi_{i}^{n}, i=1,2$ in $D^{(n)}$. In fact,

$$
\left(\xi_{1}^{n}\right)^{*} \xi_{1}^{n}+\left(\xi_{2}^{n}\right)^{*} \xi_{2}^{n}=1, \quad \xi_{1}^{n}\left(\xi_{1}^{n}\right)^{*}+\xi_{2}^{n}\left(\xi_{2}^{n}\right)^{*}=1 .
$$

Furthermore, we can choose $\xi_{1}^{n}$ and $\xi_{2}^{n}$ to be smooth functions.

Proof. On $D^{(0)}$ we get a frame from $\xi_{1}:=1, \xi_{2}:=0$. For $n \neq 0$, let $U, V$ be small neighbourhoods of $\left[0, \frac{1}{2}\right]$ and $\left[\frac{1}{2}, 1\right]$, respectively, and $f_{1}, f_{2}$ a partition of unity subordinate to $U, V$. Set $\chi_{i}=f_{i} / \sqrt{f_{1}^{2}+f_{2}^{2}}$; then $\chi_{1}^{2}+\chi_{2}^{2}=1$.

We define $\xi_{1}^{n}$ on $U \times S^{1}$ by setting $\xi_{1}^{n}(x, y)=\chi_{1}(x)$. Choosing $U$ and $V$ small enough, $\xi_{1}$ may be assumed to vanish with all derivatives on the boundary of a fundamental domain, and using the equation (6), $\xi_{1}^{n}$ can then be extended to an element of $D^{(n)}$.

A similar process can be applied to another fundamental domain and $\chi_{2}$ to obtain $\xi_{2}^{n}$. An easy computation on tailored fundamental domains yields

$$
\left(\xi_{i}^{n}\right)^{*} \xi_{i}^{n}=\overline{\chi_{i}} \chi_{i}, \quad \xi_{i}^{n}\left(\xi_{i}^{n}\right)^{*}=\chi_{i} \overline{\chi_{i}},
$$

and then $\chi_{1}^{2}+\chi_{2}^{2}=1$ ensures that

$$
\left(\xi_{1}^{n}\right)^{*} \xi_{1}^{n}+\left(\xi_{2}^{n}\right)^{*} \xi_{2}^{n}=1, \quad \xi_{1}^{n}\left(\xi_{1}^{n}\right)^{*}+\xi_{2}^{n}\left(\xi_{2}^{n}\right)^{*}=1 .
$$

Finally, notice that if $\chi_{i}$ is smooth, then so is $\xi_{i}^{n}$.

Recall that the Heisenberg group $H_{1}$ is the subgroup of $\mathrm{GL}_{3}(\mathbb{R})$ of the matrices

$$
M(r, s, t):=\left(\begin{array}{lll}
1 & s & t \\
0 & 1 & r \\
0 & 0 & 1
\end{array}\right), \quad \text { where } r, s, t \in \mathbb{R} .
$$

It has been proved (see [Rie89], Section 5) that the Heisenberg group acts on $D$. We use the following expression for the action:

$$
\alpha_{(r, s, t)}(F)(x, y, p)=e(-p(t+c s(x-r))) F(x-r, y-s, p) .
$$


The infinitesimal generators $\partial_{i}$, for $i=1,2,3$, are

$$
\begin{aligned}
& \partial_{1}(F)(p, x, y)=-\frac{\partial F}{\partial x}(p, x, y), \\
& \partial_{2}(F)(p, x, y)=-\frac{\partial F}{\partial y}(p, x, y)-i 2 \pi c p x F(p, x, y), \\
& \partial_{3}(F)(x, y, p)=-i 2 \pi p F(p, x, y) .
\end{aligned}
$$

Note that $\alpha_{(0,0, t)}$ is just the gauge action. As $M(0,0, t)$ is in the center, the action of $H_{1}$ preserves the grading and $\partial_{1}, \partial_{2}$ commute with $\partial_{3}$. A short calculation shows

$$
\left[\partial_{1}, \partial_{2}\right]=-c \partial_{3}, \quad\left[\partial_{1}, \partial_{3}\right]=0, \quad\left[\partial_{2}, \partial_{3}\right]=0
$$

Definition 42. We define the smooth quantum Heisenberg manifold $\mathcal{D}$ to be the subalgebra of $H_{1}$-smooth elements of $D$.

Note that $\mathscr{D}$ inherits the $\mathbb{Z}$-grading from $D$ and that $\mathscr{D}^{(1)}$ is actually a bimodule over $\mathscr{D}^{(0)}$.

Proposition 43. The smooth quantum Heisenberg manifold is a Fréchet algebra and a tame smooth GCP.

Proof. We can apply Remark 38 to see that QHMs are Fréchet algebras and smooth GCP. It remains to show that $\mathcal{D}$ is tame. By Lemma 41 , there is a frame with two elements for every $\mathscr{D}^{n}$. Denote by $\xi_{n}$ the sequence of frames ordered as in the notation on p. 508. Then point (i) of Definition 18 is clearly satisfied. It only remains to prove the polynomial growth.

Because scaling and adding do not change the equivalence-class of a family of seminorms, we may apply Poincaré-Birkoff-Witt (see [Hum78], 17.4) to see that it is enough to show that the seminorms of the form

$$
p_{n_{3}, n_{2}, n_{1}}(F):=\left\|\partial_{3}^{n_{3}} \partial_{2}^{n_{2}} \partial_{1}^{n_{1}}(F)\right\|_{\infty}, \quad F \in \mathcal{D},
$$

yield polynomially increasing sequences on $\xi_{n}$. We show that there is a constant $C$ (which depends on $n_{1}, n_{2}$ and $n_{3}$ but not on $n$ ) such that

$$
p_{n_{3}, n_{2}, n_{1}}\left(\xi_{n}\right) \leqslant C(1+n)^{n_{3}+n_{2}} .
$$

Trivializing over $U$ or $V, \xi_{n}$ restricts either to $\chi_{1}$ or $\chi_{2}$ (compare Lemma 41). We estimate over $U$ in the first case:

$$
\left\|p_{n_{3}, n_{2}, n_{1}} \xi_{n}\right\|=\left\|\partial_{3}^{n_{3}} \partial_{2}^{n_{2}} \partial_{1}^{n_{1}} \chi_{1}\right\| \leqslant C n^{n_{3}}\left\|\partial_{2}^{n_{2}} \chi_{1}^{\left(n_{1}\right)}\right\| \leqslant C^{\prime} n^{n_{3}}\left\|(n x)^{n_{2}} \chi_{1}^{\left(n_{1}\right)}\right\|
$$

since $\chi_{1}^{\left(n_{1}\right)}$ does not depend on $y$. The case of $\chi_{2}$ is treated similarly.

Hence, applying Theorem 36 to kk and HP, we get 
Theorem 44. There is a commutative diagram

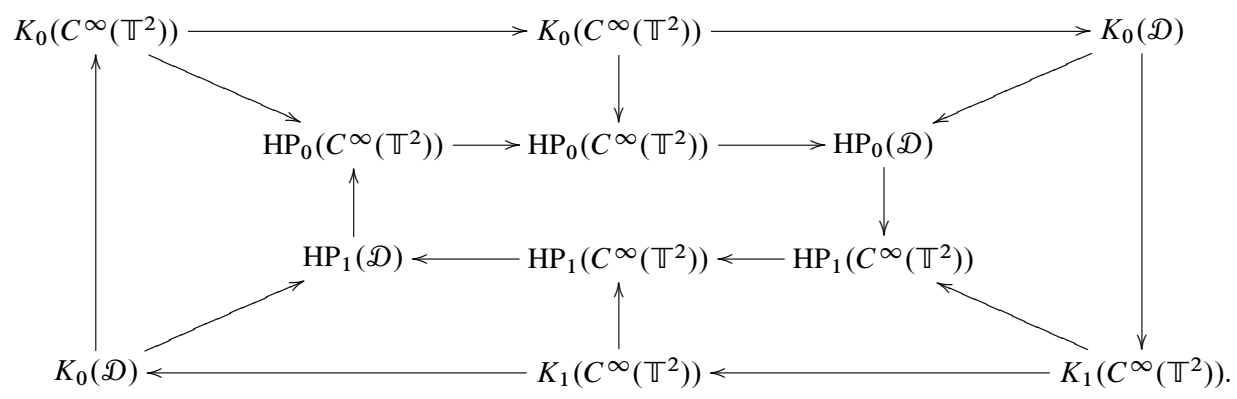

\section{References}

[AEE98] B. Abadie, S. Eilers, and R. Exel, Morita equivalence for crossed products by Hilbert $C^{*}$-bimodules. Trans. Amer. Math. Soc. 350 (1998), 3043-3054. Zbl 0899.46053 MR 1467459

[Bla98] B. Blackadar, K-theory for operator algebras. Math. Sci. Res. Inst. Publ. 5, 2nd ed., Cambridge University Press, Cambridge 1998. Zbl 0913.46054 MR 1656031

[Cun83] J. Cuntz, Generalized homomorphisms between $C^{*}$-algebras and $K K$-theory. In Dynamics and processes (Bielefeld, 1981), Lecture Notes in Math. 1031, Springer, Berlin 1983, 31-45. Zbl 0561.46034 MR 733641

[Cun84] J. Cuntz, $K$-theory and $C^{*}$-algebras. In Algebraic $K$-theory, number theory, geometry and analysis (Bielefeld, 1982), Lecture Notes in Math. 1046, Springer, Berlin 1984, 55-79. Zbl 0548.46056 MR 750677

[Cun97] J. Cuntz, Bivariante $K$-Theorie für lokalkonvexe Algebren und der Chern-ConnesCharakter. Doc. Math. 2 (1997), 139-182. Zbl 0920.19004 MR 1456322

[Cun05] J. Cuntz, Bivariant $K$-theory and the Weyl algebra. K-Theory 35 (2005), 93-137. Zbl 1111.19003 MR 2240217

[CMR07] J. Cuntz, R. Meyer, and J. M. Rosenberg, Topological and bivariant Ktheory. Oberwolfach Semin. 36, Birkhäuser Verlag, Basel 2007. Zbl 1139.19001 MR 2340673

[CST04] J. Cuntz, G. Skandalis, and B. Tsygan, Cyclic homology in non-commutative geometry. Encyclopaedia Math. Sci. 121, Springer-Verlag, Berlin 2004. Zbl 1045.46043 MR 2052770

[CT06] J. Cuntz and A. Thom, Algebraic $K$-theory and locally convex algebras. Math. Ann. 334 (2006), 339-371. Zbl 1095.19003 MR 2207702

[Gab13] O. Gabriel, K-theory, cyclic cohomology and pairings for quantum Heisenberg manifolds. J. Noncommut. Geom. 7 (2013), 301-333.

[GG10] O. Gabriel and M. Grensing, Exact sequences for locally convex subalgebras of Pimsner algebras with an application to quantum Heisenberg manifolds. Preprint 2010. arXiv:1011.6238 
[VFGB00] J. M. Gracia-Bondía, J. C. Várilly, and H. Figueroa, Elements of noncommutative geometry. Birkhäuser Adv. Texts, Birkhäuser, Boston 2001. Zbl 0958.46039 MR 1789831

[Gre11] M. Grensing, Universal cycles and homological invariants of locally convex algebras. J. Funct. Anal. 263 (2012), 2170-2204. Zbl 06104922 MR 2964680

[Hum78] J. E. Humphreys, Introduction to Lie algebras and representation theory. Graduate Texts in Math. 9, Springer-Verlag, New York 1978. Zbl 0447.17001 MR 499562

[Kat03] T. Katsura, A construction of $C^{*}$-algebras from $C^{*}$-correspondences. In Advances in quantum dynamics, Contemp. Math. 335, Amer. Math. Soc., Providence, RI, 2003, 173-182. Zbl 1049.46038 MR 2029622

[Kat04] T. Katsura, On $C^{*}$-algebras associated with $C^{*}$-correspondences. J. Funct. Anal. 217 (2004), 366-401. Zbl 1067.46054 MR 2102572

[KS97] M. Khoshkam and G. Skandalis, Toeplitz algebras associated with endomorphisms and Pimsner-Voiculescu exact sequences. Pacific J. Math. 181 (1997), 315-331. Zbl 0885.46053 MR 1486534

[Nes88] R. Nest, Cyclic cohomology of crossed products with ZZ. J. Funct. Anal. 80 (1988), 235-283. Zbl 0658.46054 MR 961899

[Pim97] M. V. Pimsner, A class of $C^{*}$-algebras generalizing both Cuntz-Krieger algebras and crossed products by $\mathbb{Z}$. In Free probability theory, Fields Inst. Commun. 12, Amer. Math. Soc., Providence, RI, 1997, 189-212. Zbl 0871.46028 MR 1426840

[PV80] M. Pimsner and D. Voiculescu, Exact sequences for $K$-groups and Ext-groups of certain cross-product $C^{*}$-algebras. J. Operator Theory 4 (1980), 93-118. Zbl 0474.46059 MR 587369

[Rie89] M. A. Rieffel, Deformation quantization of Heisenberg manifolds. Comm. Math. Phys. 122 (1989), 531-562. Zbl 0679.46055 MR 1002830

Received April 7, 2011

O. Gabriel, Université Denis Diderot Paris 7 - Institut de Mathématiques de Jussieu Équipe algèbres d'opérateurs, 175, rue du Chevaleret, 75013 Paris, France

E-mail: gabriel@math.jussieu.fr

M. Grensing, Université Denis Diderot Paris 7 - Institut de Mathématiques de Jussieu Équipe algèbres d'opérateurs, 175, rue du Chevaleret, 75013 Paris, France

E-mail: mgren@math.jussieu.fr 